\title{
Weak-measurement-induced phases and dephasing: Broken symmetry of the geometric phase
}

\author{
Kyrylo Snizhko $\odot,{ }^{1,2}$ Nihal Rao $\odot,{ }^{1, *}$ Parveen Kumar, ${ }^{1}$ and Yuval Gefen ${ }^{1}$ \\ ${ }^{1}$ Department of Condensed Matter Physics, Weizmann Institute of Science, Rehovot 76100, Israel \\ ${ }^{2}$ Institute for Quantum Materials and Technologies, Karlsruhe Institute of Technology, 76021 Karlsruhe, Germany
}

(Received 29 June 2020; accepted 10 September 2021; published 18 October 2021)

\begin{abstract}
Coherent steering of a quantum state, induced by a sequence of weak measurements, has become an active area of theoretical and experimental study. For a closed steered trajectory, the underlying phase factors involve both geometrical and dynamical terms. Furthermore, considering the reversal of the order of the measurement sequence, such a phase comprises a symmetric and an antisymmetric term. Superseding common wisdom, we show that the symmetric and the antisymmetric components do not correspond to the dynamical and geometrical parts, respectively. Addressing a broad class of measurement protocols, we further investigate the dependence of the induced phases on the measurement parameters (e.g., the measurement strength). We find transitions between different topologically distinct sectors, defined by integer-valued winding numbers, and show that the transitions are accompanied by diverging dephasing. We propose experimental protocols to observe these effects.
\end{abstract}

DOI: 10.1103/PhysRevResearch.3.043045

\section{INTRODUCTION}

Geometrical phases are a cornerstone of modern physics [1]. The work of Berry [2] provided a unifying language that is key to understanding disparate phenomena including the quantum Hall effect $[3,4]$ and topological insulators [5], sheds light on some features of graphene [6,7], and provides the basis for geometric [8,9] and topological [10] quantum computation platforms. Geometrical phases can be induced not only by means of adiabatic [2] or nonadiabatic [11,12] Hamiltonian manipulation, but also as a result of a sequence of projective measurements [13-15]. In that case, the phase is called the Pancharatnam phase, after the physicist who discovered it in the context of classical optics [16]. Recently, the possibility of inducing geometrical phases by weak measurements [17,18] was demonstrated experimentally [19]. Moreover, a topological transition in the behavior of the geometrical phase as a function of the measurement strength has been predicted theoretically [20].

Here we outline a general framework for treating measurement-induced phase factors and apply it to a broad class of measurements. Our analysis addresses the nature of the phase accumulated during a sequence of weak measurements, a generalization of the concept of the geometrical

\footnotetext{
*Present address: Arnold Sommerfeld Center for Theoretical Physics, University of Munich, Theresienstraße 37, 80333 München, Germany and Munich Center for Quantum Science and Technology, Schellingstraße 4, 80799 München, Germany.

Published by the American Physical Society under the terms of the Creative Commons Attribution 4.0 International license. Further distribution of this work must maintain attribution to the author(s) and the published article's title, journal citation, and DOI.
}

Pancharatnam phase in the case of strong (projective) measurements. In previous investigations, measurement-induced phase factors were of a purely geometric origin $[13,19,20]$. In the presence of an additional Hamiltonian acting on the measured system, an additional dynamical component appears [14]. We demonstrate that weak-measurement-induced phases generically involve both geometrical and dynamical components even in the absence of an additional Hamiltonian. This fact went unnoticed in earlier studies that focused on restricted classes of measurements. Quantum measurements are characterized by Kraus operators describing the consequent backaction [21-23]. While previous works focused on the case of Hermitian Kraus operators, in the more general case of non-Hermitian Kraus operators, considered here, not all of measurement-induced phases can qualify as geometrical.

We also investigate the behavior of the phase with respect to reversing the order of the measurement sequence. The fact that there is no definite symmetry with respect to such a reversal implies that measurement-induced phases can be split into symmetric and antisymmetric components. Interestingly, and superseding the common structure of phases generated by conventional adiabatic Hamiltonian dynamics, the symmetric and antisymmetric components do not coincide with the dynamical and geometrical components, respectively.

These general insights are then demonstrated through the analysis of specific measurement protocols. We study two types of such protocols: one which involves postselection and a second which involves averaging over all measurement outcomes (i.e., no postselection). Postselection refers to selecting experimental runs that yield a desired set of measurement readouts. An important quantity here is the postselection probability, i.e., the probability to have a predesignated readout sequence. Concerning the other protocol, one averages the readout-sequence-dependent phase over many experimental 
runs, which gives rise to a suppression factor also known as dephasing.

Finally, we focus on topological transitions in the context of measurement-induced phases. Previously, such transitions were predicted for a restricted class of measurements with Hermitian backaction [20]. Here we show that such transitions may still take place when the backaction is non-Hermitian. Under such general backaction, multiple distinct topological sectors exist, forming a rich phase diagram. Transitions between such sectors are marked by (i) a vanishing probability of the corresponding postselected sequence (the case of postselective protocols) and (ii) diverging dephasing (the case of averaging protocols). ${ }^{1}$ We also propose and analyze experimental setups that may test our predictions.

The paper is organized as follows. Section II first recaps the theory of generalized quantum measurements. We then define measurement-induced phases and discuss their classifications into dynamical/geometrical and symmetric/antisymmetric terms and the relation between these two classifications. In Sec. III we specify the measurements and protocols to be employed. We derive and analyze analytic expressions for the induced phases, the postselection probabilities, and the dephasing factors. Section IV presents a mostly numerical analysis of the topological transitions vis-à-vis postselective protocols. Section V presents a similar analysis for the phase-averaging protocol. In Sec. VI we discuss possible experimental implementations. A summary and conclusions are presented in Sec. VII. Three Appendixes of technical nature are included. Appendix A presents a justification of our choice of scaling of the measurement parameters with the number of measurements (cf. Sec. III). Appendix B provides an analytic derivation of the critical line of topological transitions in the postselective protocol (cf. Sec. IV). Appendix C provides the justification for the averaged phase detection scheme proposed in Sec. VI.

\section{WEAK-MEASUREMENT-INDUCED PHASES: DEFINITIONS AND GENERAL ANALYSIS}

In this section we present a general analysis of measurement-induced phase factors. We briefly recall the theory of generalized quantum measurements in Sec. II A. We then proceed to define postselected and averaged measurement-induced phases in Sec. II B. We analyze various characteristics of these phases and discuss possible classifications thereof in Sec. II C.

\section{A. Theory of generalized measurements}

Describing a conventional projective measurement in quantum theory requires a Hermitian observable $\mathcal{O}$ of the measured system. The observable has a set of eigenstates labeled by its eigenvalues $\lambda, \mathcal{O}|\lambda\rangle=\lambda|\lambda\rangle$. A projective measurement yields a readout $r$ which corresponds to one of the eigenvalues $\lambda$. If a readout $r=\lambda$ is obtained, the system state becomes $\left|\psi^{(r=\lambda)}\right\rangle=\mathcal{P}_{\lambda}|\psi\rangle$, where $|\psi\rangle$ is the system

\footnotetext{
${ }^{1} \mathrm{~A}$ short exposition of our results pertaining to topological transitions in the averaging protocols can be found in Ref. [24].
}

state before the measurement and $\mathcal{P}_{\lambda}=|\lambda\rangle\langle\lambda|$ is the projector onto the corresponding eigenstate of $\mathcal{O}$ (generalization to the case of a degenerate spectrum is straightforward). Note that $\left|\psi^{(r=\lambda)}\right\rangle$ is not normalized. The probability of the projective measurement yielding $r=\lambda$ is $p_{r=\lambda}=|\langle\lambda \mid \psi\rangle|^{2}=$ $\left\langle\psi^{(r=\lambda)} \mid \psi^{(r=\lambda)}\right\rangle$.

Generalized measurement [21-23] is an extension of the orthodox concept of projective measurement. The extension is based on treating the detector as an additional quantummechanical object. The measurement is then conceptually described as a two-step protocol: (i) The system is coupled to the detector and then decoupled and (ii) the detector is measured projectively. The strength of the interaction between the system and the detector defines the measurement strength.

The formal description of such a protocol is as follows. Let the system initial state be $|\psi\rangle$ in the system Hilbert space $\mathcal{H}_{\mathrm{s}}$ and the detector initial state be $\left|D_{i}\right\rangle$ in the detector Hilbert space $\mathcal{H}_{\mathrm{d}}$. During the first step, they interact via Hamiltonian $H_{\mathrm{s}-\mathrm{d}}(t)$, which vanishes outside the interval $t \in[0, T]$ (i.e., the interaction Hamiltonian is switched on at $t=0$ and off at $t=T$ ). In the second step, the detector is measured projectively with readouts corresponding to some basis $\{|r\rangle\}$ in the detector's Hilbert space. The outcome of the first step is the evolution of the system-detector state

$$
\begin{aligned}
|\psi\rangle\left|D_{i}\right\rangle & \rightarrow \mathcal{T} \exp \left(-i \int_{0}^{T} H_{\mathrm{s}-\mathrm{d}}(t) d t\right)|\psi\rangle\left|D_{i}\right\rangle \\
& =\sum_{r}\left|\psi^{(r)}\right\rangle|r\rangle,
\end{aligned}
$$

where $\mathcal{T}$ stands for time ordering; the last equality represents a decomposition that can be performed for any pure state in the system-detector Hilbert space $\mathcal{H}_{\mathrm{s}} \otimes \mathcal{H}_{\mathrm{d}}$. For a specific system-detector Hamiltonian $H_{\mathrm{s}-\mathrm{d}}(t)$ and detector's initial state $\left|D_{i}\right\rangle$, the resulting system state can be written as [22]

$$
\left|\psi^{(r)}\right\rangle=\mathcal{M}^{(r)}|\psi\rangle
$$

where the Kraus operators

$$
\mathcal{M}^{(r)}=\left\langle r\left|\mathcal{T} \exp \left(-i \int_{0}^{T} H_{\mathrm{s}-\mathrm{d}}(t) d t\right)\right| D_{i}\right\rangle
$$

represent the measurement's nonlocal backaction, following the detector's projective readout. The probability of obtaining a specific readout $r$ is

$$
p_{r}=\left\langle\psi^{(r)} \mid \psi^{(r)}\right\rangle=\left\langle\psi\left|\mathcal{M}^{(r) \dagger} \mathcal{M}^{(r)}\right| \psi\right\rangle .
$$

Conservation of probability $\sum_{r} p_{r}=1$ independently of the system's initial state $|\psi\rangle$ implies

$$
\sum_{r} \mathcal{M}^{(r) \dagger} \mathcal{M}^{(r)}=\mathbb{I},
$$

where $\mathbb{I}$ is the identity operator acting in the system's Hilbert space. This is the only restriction on the Kraus operators, which otherwise are arbitrary. ${ }^{2}$

\footnotetext{
${ }^{2}$ Anticipating the use of multiple measurements in our protocols below, we comment on the relation between the dynamics of a system under measurement and the dynamics of an open system. Indeed, a
} 
One thus sees that a description of a generalized quantum measurement does not require microscopic modeling of the detector. It is sufficient to specify the set of possible readouts $\{r\}$ and the corresponding Kraus operators $\mathcal{M}^{(r)}$ acting on the system. The Kraus operators are thus the analogs of the projection operators $\mathcal{P}_{\lambda}$ that describe the backaction of a projective measurement. If the Kraus operators $\mathcal{M}^{(r)}$ are Hermitian and $\left(\mathcal{M}^{(r)}\right)^{2}=\mathcal{M}^{(r)}$ then they can be interpreted as projectors $\mathcal{P}_{r}$ and the generalized measurement scheme reduces to the projective measurement scenario.

It is important to realize the following difference between projective and generalized measurements. In a projective measurement, a specific readout $r=\lambda$ always corresponds to the system being collapsed onto a specific state $|\lambda\rangle \propto \mathcal{P}_{\lambda}|\psi\rangle$ following the measurement. This is not necessarily so for generalized measurements. Indeed, knowing the initial state $|\psi\rangle$ and the readout $r$, one finds the system state after the measurement according to Eq. (2). However, knowing the measurement readout alone does not suffice for the determination of $\left|\psi^{(r)}\right\rangle$. An example of the generalized measurement backaction is presented in Fig. 1.

It is instructive to understand how the system observable measured, the system-detector interaction Hamiltonian, and the Kraus operators are related. For that, consider a simple canonical example of the system-detector Hamiltonian

$$
H_{\mathrm{s}-\mathrm{d}}(t)=\lambda \theta_{[0, T]}(t) \mathcal{O} \otimes A^{(\mathrm{d})},
$$

where $\mathcal{O}$ is the system observable measured, $A^{(\mathrm{d})}$ is an operator acting in the detector Hilbert space $\mathcal{H}_{\mathrm{d}}, \lambda$ is the coupling strength, and

$$
\theta_{[0, T]}(t)= \begin{cases}0 & \text { if } t<0 \text { or } t>T \\ 1 & \text { if } 0<t<T .\end{cases}
$$

Note that the same measurement setup (the same system and detector, same detector initial state, and same detector readout basis) can be used with different observables $\mathcal{O}$, particularly with observables of the form $R^{-1} \mathcal{O} R$, where $R$ is a unitary

detector can be regarded as the system's environment, putting the measurement-induced dynamics into the framework of open quantum systems. However, the formal analogy is incomplete without emphasizing a key difference between the measurement-induced and open-system dynamics. The standard treatment of an open system does not consider modulating the system-environment coupling or directly controlling the environment in the course of experiment. As a result, a classification into Markovian and non-Markovian open system dynamics (depending on the environment properties) arises. In contrast, measurement-based dynamics includes the following aspects: modulating system-detector couplings to separate distinct measurements, reading out the detector, and preparing the detector in a specific initial state before the next measurement. As a result, the measurement-induced dynamics is non-Markovian on the scale of a single measurement (as the detector does not relax and thus preserves memory of the system's previous states during the measurement), but is Markovian beyond the single-measurement timescale (the detector is read out and initialized before each measurement; therefore, the result of a measurement depends only on the system state at the beginning of the measurement and not on the previous system states/measurement readouts).

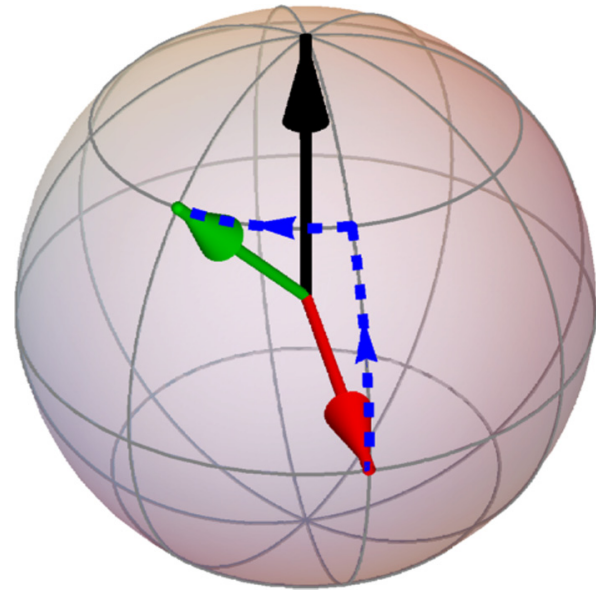

FIG. 1. Backaction of a generalized measurement. After a projective measurement of the $S_{z}$ component of spin $\frac{1}{2}$ that yields a readout $S_{z}=+\frac{1}{2}$, the initial state (red arrow) becomes aligned with the north pole of the Bloch sphere (black arrow). A generalized measurement's backaction does not necessarily align the state with an eigenstate of the measured observable; it only pulls the state towards the north pole and may also rotate it around the $z$ axis (green arrow). These two effects of the backaction are illustrated by blue dashed lines. Such a backaction appears in the measurement protocol we consider in Sec. III A.

rotation in the system Hilbert space. The backaction of the measurement then changes as

$$
\mathcal{M}^{(r)} \rightarrow R^{-1} \mathcal{M}^{(r)} R .
$$

Equation (8) defines a family of measurements of the same class. Modifying the system-detector interaction, e.g., by selecting an observable $\mathcal{O}^{\prime}$ with a different spectrum, modifies the nature of the measurement at hand, thus introducing a different measurement class. The measurement class may be altered even more drastically, e.g., by keeping the same observable but taking a different detector (with different operator $A^{(\mathrm{d})}$, different initial state $\left|D_{i}\right\rangle$, different readout basis $\{|r\rangle\}$, or even different Hilbert space $\mathcal{H}_{\mathrm{d}}$ ). Thus, measurements of different classes can apply to the same observable $\mathcal{O}$ but yield drastically different backactions or even have different sets of possible readouts $r$. In principle, nothing prevents us from implementing measurements of different classes on a given system at different times.

\section{B. Measurement-induced phases}

Consider a sequence of $N+1$ distinct measurements performed on a quantum system. Each measurement is fully characterized by a set of Kraus operators $\left\{\mathcal{M}_{k}^{\left(r_{k}\right)}\right\}$, where $k=$ $1, \ldots, N+1$ is the measurement number and $r_{k}$ is the measurement readout. These can be measurements of the same class yet measuring different system observables (e.g., same strength measurements of the spin projection onto different directions), in which case

$$
\mathcal{M}_{k}^{\left(r_{k}\right)}=R_{k}^{-1} M^{\left(r_{k}\right)} R_{k},
$$

where $R_{k}$ is a unitary rotation in the system Hilbert space and the Kraus operator $M^{(r)}$ does not depend on $k$. This is 
the case for the example considered in Ref. [20] and for the one discussed in Sec. III. However, in the present section we keep the analysis general. In particular, we allow for situations where the Kraus operators for different measurements are not simply related and even the number of possible readout values $r_{k}$ for different measurements can be different.

Consider a system prepared in a certain initial state $\left|\psi_{0}\right\rangle$. Assuming knowledge of all readouts $\left\{r_{k}\right\}$ of the measurement sequence, the system state traverses a sequence of states

$$
\left|\psi_{k}\right\rangle=\mathcal{M}_{k}^{\left(r_{k}\right)} \cdots \mathcal{M}_{2}^{\left(r_{2}\right)} \mathcal{M}_{1}^{\left(r_{1}\right)}\left|\psi_{0}\right\rangle .
$$

We choose the last measurement to be projective and postselect the final state such that it coincides with the system's initial state, i.e., $\mathcal{M}_{N+1}^{(0)}=\mathcal{P}_{0}=\left|\psi_{0}\right\rangle\left\langle\psi_{0}\right|$. Then the system state after completing the entire sequence of measurements

$$
\left|\psi_{N+1}\right\rangle=\left|\psi_{0}\right\rangle\left\langle\psi_{0}\left|\mathcal{M}_{N}^{\left(r_{N}\right)} \cdots \mathcal{M}_{1}^{\left(r_{1}\right)}\right| \psi_{0}\right\rangle
$$

differs from the initial state by a factor

$$
\left\langle\psi_{0}\left|\mathcal{M}_{N}^{\left(r_{N}\right)} \cdots \mathcal{M}_{1}^{\left(r_{1}\right)}\right| \psi_{0}\right\rangle=\sqrt{P_{\left\{r_{k}\right\}}} e^{\left.i \chi_{\left[r_{k}\right\}}\right\}} .
$$

This factor has two components: $P_{\left\{r_{k}\right\}}$ is the probability to observe the readout sequence $\left\{r_{1}, \ldots, r_{N}, r_{N+1}=0\right\}$, while

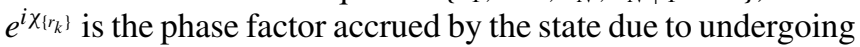
measurement-induced evolution. In what follows we refer to $\chi_{\left\{r_{k}\right\}}$ as the postselected measurement-induced phase. ${ }^{3}$ In the case where all the measurements are projective, $\chi_{\left\{r_{k}\right\}}$ reduces to the projective-measurement-induced Pancharatnam phase $[13,15,16]$.

It is also possible to define the averaged measurementinduced phase through

$$
\begin{aligned}
e^{2 i \bar{\chi}-\alpha} & =\sum_{\left\{r_{k}\right\}}\left(\left\langle\psi_{0}\left|\mathcal{M}_{N}^{\left(r_{N}\right)} \cdots \mathcal{M}_{1}^{\left(r_{1}\right)}\right| \psi_{0}\right\rangle\right)^{2} \\
& =\sum_{\left\{r_{k}\right\}} P_{\left\{r_{k}\right\}} e^{2 i \chi_{\left.r_{k}\right\}}},
\end{aligned}
$$

\footnotetext{
${ }^{3}$ It is known that the phase of an individual quantum state can be chosen arbitrarily, which is often referred to as the gauge freedom. The phase defined here is gauge invariant. In order to understand this, consider the two potential sources of non-gauge-invariance. One source is the freedom of choosing the phase of the initial state $\left|\psi_{0}\right\rangle \rightarrow e^{i \phi}\left|\psi_{0}\right\rangle$. One immediately sees that this freedom does not affect Eq. (12); the physical reason is that, just as in Ref. [2], the phase is defined via comparison of the state after some process with the system's initial state. The second source is the gauge freedom associated with choosing the phase of the detector states $\left|D_{i}\right\rangle$ and $|r\rangle$, which would affect the phase of the Kraus operators $\mathcal{M}^{(r)}$ [cf. Eq. (3)] . This freedom is however eliminated by demanding that when the system does not interact with detectors $\left[H_{\mathrm{s}-\mathrm{d}}(t) \equiv 0\right]$, no phase is accumulated. In practice (cf. the experimental setups proposed in Sec. VIA), both of these theoretical sources of gauge noninvariance are made inconsequential by the structure of the interferometer, which ensures that (i) the unaffected initial state is compared to the state after a sequence of measurements, directly implementing the first theoretical argument; (ii) the detector preparation and readout are performed independently of the path taken by the particle and therefore any phases associated with $\left\langle r \mid D_{i}\right\rangle$ "cancel out" in the interference pattern.
}

where the sum runs over all possible readout sequences $\left\{r_{k}\right\}$ (such that $r_{N+1}=0$ ). Here $\bar{\chi}$ is the averaged measurementinduced phase. The real parameter $\alpha \geqslant 0$ has a mixed meaning. It characterizes the dephasing due to averaging over various measurement readout sequences $\left\{r_{k \leqslant N}\right\}$; at the same time, the finite probability of obtaining $r_{N+1}=0$ in the last projective measurement also contributes to $\alpha$. Hereafter we will refer to $\alpha$ as the dephasing parameter and to $e^{-\alpha}$ as the dephasing-induced suppression factor.

One may wonder why the averaged phase is defined through the averaging of $e^{\left.2 i x_{r_{k}}\right\}}$ in Eq. (13) and not through

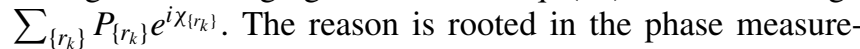
ment procedure, discussed in detail in Sec. VI. Here we only briefly explain the idea behind the procedure of observing the averaged phase. Different readout sequences $\left\{r_{k}\right\}$ correspond to mutually orthogonal states of detectors employed throughout the sequence of measurements. At the same time, measuring a phase requires interference between two states, e.g., the unmeasured and the measured states. For measuring the phase corresponding to a postselected sequence $\left\{r_{k}\right\}$, one can use an interferometer, in one arm of which the system (spin of the flying particle) is measured and in the other it is not [cf. Fig. 12(a)]. If the initial state of all detectors coincides with the state corresponding to the postselected readout sequence, the interference pattern exhibits a nonvanishing visibility, which allows for measuring $\chi_{\left\{r_{k}\right\}}$. This may work for one particular postselected readout sequence. However, averaging requires the consideration of numerous readout sequences, the vast majority of which are orthogonal to the sequence of null readouts, expected when no systemdetector coupling is present (i.e., when the interfering particle goes through the reference interferometer arm which does not involve coupling to detectors). To facilitate averaging over different readout sequences, one needs to couple detectors to both arms of the interferometer [cf. Fig. 12(b)]. This facilitates maintaining coherence between the two arms independently of the measurement readouts. In other words, readouts do not constitute a "which path" measurement [25,26]. We design the couplings such that traversing one arm of the interferometer or the other, the system accumulates opposite phases $e^{\left.i \chi_{\left.r_{k}\right]}\right]}$ and

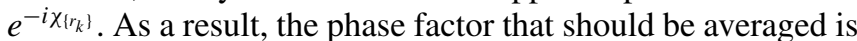
$e^{2 i \chi_{\left.i r_{k}\right\}} .}$

\section{Classification of measurement-induced phases}

In Sec. II B we defined postselected (12) and averaged (13) measurement-induced phases. Here we investigate their separation into dynamical and geometrical components and their symmetry properties with respect to reversing the order of the measurement sequence.

Consider a specific readout sequence $\left\{r_{k}\right\}$. The system traverses a trajectory in the Hilbert space corresponding to states $\left|\psi_{k}\right\rangle$, defined in Eq. (10). It is known that any quantum system traversing a trajectory in the Hilbert space accumulates a Pancharatnam phase $[13,15,16]$

$$
\arg \left\langle\psi_{0}\left|\mathcal{P}_{N} \cdots \mathcal{P}_{1}\right| \psi_{0}\right\rangle,
$$

where arg denotes the argument of a complex number and $\mathcal{P}_{k}=\left|\psi_{k}\right\rangle\left\langle\psi_{k}\right| /\left\langle\psi_{k} \mid \psi_{k}\right\rangle$ are the projectors onto the respective intermediate states. Does Eq. (14) coincide with $\chi_{\left\{r_{k}\right\}}$ ? In 
general, Pancharatnam's geometrical phase (14) does not coincide with $\chi_{\left\{r_{k}\right\}}$ [Eq. (12)], implying that the latter has geometrical and nongeometric (also known as dynamical) components.

One can articulate a simple condition for $\chi_{\left\{r_{k}\right\}}$ to be purely geometrical. If the Kraus operators used in the measurement sequence are Hermitian $\left(\mathcal{M}_{k}^{\left(r_{k}\right) \dagger}=\mathcal{M}_{k}^{\left(r_{k}\right)}\right)$ and positive semidefinite $\left(\left\langle\psi\left|\mathcal{M}_{k}^{\left(r_{k}\right)}\right| \psi\right\rangle \geqslant 0\right.$ for any $\left.|\psi\rangle\right)$, then $\left\langle\psi_{k+1} \mid \psi_{k}\right\rangle=\left\langle\psi_{k}\left|\mathcal{M}_{k+1}^{\left(r_{k+1}\right) \dagger}\right| \psi_{k}\right\rangle=\left\langle\psi_{k}\left|\mathcal{M}_{k+1}^{\left(r_{k+1}\right)}\right| \psi_{k}\right\rangle \geqslant 0$. This implies that

$$
\begin{aligned}
& \arg \left\langle\psi_{0}\left|\mathcal{M}_{N}^{\left(r_{N}\right)} \cdots \mathcal{M}_{1}^{\left(r_{1}\right)}\right| \psi_{0}\right\rangle=\arg \left\langle\psi_{0} \mid \psi_{N}\right\rangle \\
& =\arg \left\langle\psi_{0} \mid \psi_{N}\right\rangle+\arg \prod_{k=0}^{N-1}\left\langle\psi_{k+1} \mid \psi_{k}\right\rangle \\
& \quad=\arg \left\langle\psi_{0}\left|\mathcal{P}_{N} \cdots \mathcal{P}_{1}\right| \psi_{0}\right\rangle,
\end{aligned}
$$

so that $\chi_{\left\{r_{k}\right\}}$ coincides with the Pancharatnam phase. ${ }^{4}$ Generically, however, the Kraus operators are not Hermitian. Then $\left\langle\psi_{k+1} \mid \psi_{k}\right\rangle=\left\langle\psi_{k}\left|\mathcal{M}_{k+1}^{\left(r_{k+1}\right) \dagger}\right| \psi_{k}\right\rangle$ is not constrained to be real (not to mention non-negative), Eq. (15) does not hold, and the phases $\chi_{\left\{r_{k}\right\}}$ are not uniquely determined by the measurementinduced state trajectory (although knowledge of the trajectory together with the measurement parameters clearly does determine the phase). We call the difference

$\chi_{\left\{r_{k}\right\}}^{(\text {dyn })}=\arg \left\langle\psi_{0}\left|\mathcal{M}_{N}^{\left(r_{N}\right)} \cdots \mathcal{M}_{1}^{\left(r_{1}\right)}\right| \psi_{0}\right\rangle-\arg \left\langle\psi_{0}\left|\mathcal{P}_{N} \cdots \mathcal{P}_{1}\right| \psi_{0}\right\rangle$

the dynamical component of the measurement-induced phase.

This consideration implies that the averaged phase $\bar{\chi}$ too may not be assigned the meaning of a purely geometrical phase. However, in the case of averaging, our discussion below does not provide an algorithm for separating the phase into geometrical and dynamical components.

For adiabatic Hamiltonian evolution driven by a Hamiltonian $H(t \in[0, T])$, the phase factor accumulated in the course of the evolution can be split into dynamical and geometrical (Berry) components [2]. These components present the following property: Evolving the system in the opposite direction, $H(t) \rightarrow H(T-t)$, keeps the dynamical component unchanged and reverses the sign of the Berry component. This has been the basis for separating dephasing in open systems undergoing adiabatic evolution into dynamical and geometrical components [27-32]. Evidently, it is of interest to investigate the behavior of measurement-induced phases under reversing the measurement sequence.

Consider the same protocol as in Sec. II B but with the intermediate measurements executed in the opposite order. The postselected phase $\chi_{\left\{r_{k}\right\}}$ [cf. Eq. (12)] is then defined through $\left\langle\psi_{0}\left|\mathcal{M}_{1}^{\left(r_{1}\right)} \ldots \mathcal{M}_{N}^{\left(r_{N}\right)}\right| \psi_{0}\right\rangle=\left\langle\psi_{0}\left|\mathcal{M}_{N}^{\left(r_{N}\right) \dagger} \cdots \mathcal{M}_{1}^{\left(r_{1}\right) \dagger}\right| \psi_{0}\right\rangle^{*}$. For Hermitian Kraus operators, the last expression is equal to $\left\langle\psi_{0}\left|\mathcal{M}_{N}^{\left(r_{N}\right)} \ldots \mathcal{M}_{1}^{\left(r_{1}\right)}\right| \psi_{0}\right\rangle^{*}$, meaning that the phase $\chi_{\left\{r_{k}\right\}}$ reverses its sign while the probability of the readout sequence

\footnotetext{
${ }^{4}$ Note that this Pancharatnam phase is determined by the system's intermediate states and not by the measurement directions. The latter coincide with the former only for projective measurements.
}

$P_{\left\{r_{k}\right\}}$ is unchanged [cf. Eq. (12)]. For general (non-Hermitian) Kraus operators, however, no simple relation exists between the direct and the reversed protocols. Moreover, as we show below, even the geometrical component of the phase $\chi_{\left\{r_{k}\right\}}$ does not possess a simple symmetry with respect to the reversal of the protocol's direction. One may then define the symmetric and the antisymmetric components of the measurement-induced phases

$$
\begin{aligned}
& \chi_{\left\{r_{k}\right\}}^{s / a}=\chi_{\left\{r_{k}\right\}}^{(d=+1)} \pm \chi_{\left\{r_{k}\right\}}^{(d=-1)}, \\
& \bar{\chi}^{s / a}=\bar{\chi}^{(d=+1)} \pm \bar{\chi}^{(d=-1)},
\end{aligned}
$$

where $\chi_{\left\{r_{k}\right\}}^{(d)}$ for the direct $(d=+1)$ and reversed $(d=-1)$ protocols are defined via

$$
\begin{aligned}
\left\langle\psi_{0}\left|\mathcal{M}_{N}^{\left(r_{N}\right)} \cdots \mathcal{M}_{1}^{\left(r_{1}\right)}\right| \psi_{0}\right\rangle & =\sqrt{P_{\left\{r_{k}\right\}}^{(d=+1)}} e^{i \chi_{\left\{r_{k}\right\}}^{(d=+1)}}, \\
\left\langle\psi_{0}\left|\mathcal{M}_{1}^{\left(r_{1}\right)} \cdots \mathcal{M}_{N}^{\left(r_{N}\right)}\right| \psi_{0}\right\rangle & =\sqrt{P_{\left\{r_{k}\right\}}^{(d=-1)}} e^{i \chi_{\left\{r_{k}\right\}}^{(d=-1)}}
\end{aligned}
$$

and the averaged phases $\bar{\chi}^{(d)}$ are defined via

$$
e^{2 i \bar{\chi}^{(d)}-\alpha^{(d)}}=\sum_{\left\{r_{k}\right\}} P_{\left\{r_{k}\right\}}^{(d)} e^{2 i \chi_{\left\{r_{k}\right\}}^{(d)}} .
$$

Similarly, we introduce the symmetric and antisymmetric components of the probabilities and the dephasing parameter:

$$
\begin{gathered}
P_{\left\{r_{k}\right\}}^{s}=\sqrt{P_{\left\{r_{k}\right\}}^{(d=+1)} P_{\left\{r_{k}\right\}}^{(d=-1)},} \\
P_{\left\{r_{k}\right\}}^{a}=\sqrt{P_{\left\{r_{k}\right\}}^{(d=+1)} / P_{\left\{r_{k}\right\}}^{(d=-1)}}, \\
\alpha^{s / a}=\alpha^{(d=+1)} \pm \alpha^{(d=-1)} .
\end{gathered}
$$

The above considerations lead to the following major conclusion: Unlike in adiabatic Hamiltonian evolution, the classification of contributions to the measurement-induced phase into symmetric vs antisymmetric does not coincide with the classification into dynamical vs geometrical contributions. An intuitive understanding of this result relies on the following observation: The intermediate states $\left|\psi_{k}\right\rangle$ for the direct and the reversed measurement sequences form different trajectories (cf. Fig. 2), implying that the geometrical phase components (14) are different in magnitude for the direct and the reversed protocols. We present an explicit illustration of this in Sec. III E 3.

\section{WEAK-MEASUREMENT-INDUCED PHASES: AN EXPLICIT EXAMPLE}

In the rest of this paper we focus on a specific measurement class (Sec. III A) and a specific set of measurement sequences that make the system's spin $\frac{1}{2}$ follow closed trajectories on the Bloch sphere (Sec. III B). In Sec. III C we present analytic expressions for the postselected measurement-induced phase under this protocol. In Sec. III D we outline a procedure that allows one to calculate the averaged phase in an efficient manner. Explicit results, pertaining to certain limiting cases (e.g., nearly projective measurements), are presented in Sec. III E. 


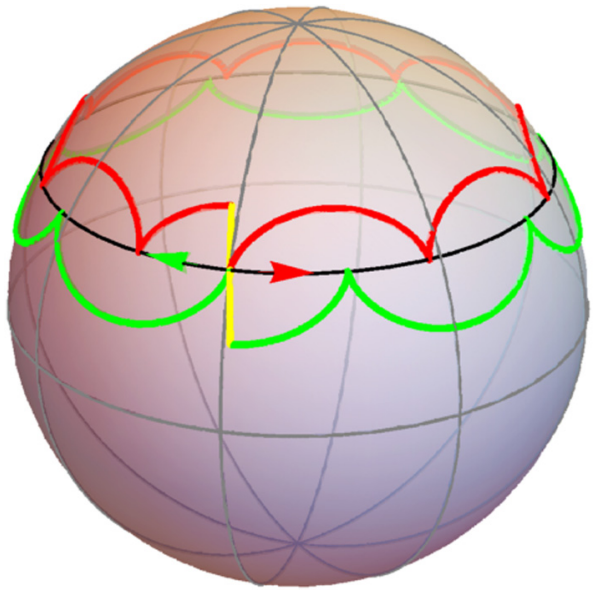

FIG. 2. State trajectories of the system for the direct and reversed measurement sequences may be drastically different. A spin- $\frac{1}{2}$ system, whose states are represented on the Bloch sphere, is subject to a sequence of generalized measurements, pertaining to the spin projections around a given parallel (black line), in accordance with the protocol described in Sec. III. All measurements of either the clockwise (red) or the counterclockwise (green) sequences are read out and postselected to have $r_{k}=0$. The yellow lines connect $\left|\psi_{N}\right\rangle$ to $\left|\psi_{0}\right\rangle$ by the shortest geodesic on the Bloch sphere. This line represents closing the trajectories by a postselected projective measurement at the end of the measurement sequence [see the discussion between Eqs. (10) and (12)]. It is known that, similar to the Berry phase, the Pancharatnam phase (14) for a spin- $\frac{1}{2}$ system may be expressed in terms of the solid angle enclosed by the closed trajectory. The trajectories under the direct and reversed measurement sequences do not subtend the same solid angle on the Bloch sphere. Their solid angles differ by sign and in absolute value. Clearly the Pancharatnam phases [see Eq. (14)] under the two time-reversed sequences are different.

\section{A. Measurement model}

Here we describe the measurement model that gives rise to the specific backaction matrices used throughout the rest of the paper. We consider a two-state system (with basis states $|0\rangle_{s}$ and $|1\rangle_{s}$ ) and a two-state detector (with basis states $|0\rangle_{D}$ and $|1\rangle_{D}$ ) with the measurement procedure being as follows. The detector is prepared in the state $\left|D_{i}\right\rangle=|0\rangle_{D}$. We choose the system-detector interaction Hamiltonian (cf. Sec. II A) to be

$$
H_{\mathrm{s}-\mathrm{d}}=-\frac{\lambda(t)}{2}\left[1-\left(\mathbf{n}^{(s)} \cdot \boldsymbol{\sigma}^{(s)}\right)\right]\left(\mathbf{n}^{(D)} \cdot \boldsymbol{\sigma}^{(D)}\right) .
$$

It is switched on during a time interval of duration $T$, i.e., $\lambda(t<0)=\lambda(t>T)=0 ; \sigma^{(s / D)}$ are the vectors of Pauli matrices $\left(\sigma_{x}, \sigma_{y}, \sigma_{z}\right)$ acting on the system/detector. The vectors

$$
\mathbf{n}^{(s / D)}=\left(\sin \theta^{(s / D)} \cos \varphi^{(s / D)}, \sin \theta^{(s / D)} \sin \varphi^{(s / D)}, \cos \theta^{(s / D)}\right)
$$

determine the system observable measured $\left(\mathbf{n}^{(s)} \cdot \boldsymbol{\sigma}^{(s)}\right)$ and the effect of the system-detector interaction on the detector state. Note that the vectors $\mathbf{n}^{(s)}$ and $\mathbf{n}^{(D)}$ are normalized, $\mathbf{n}^{(s)} \cdot \mathbf{n}^{(s)}=$ $\mathbf{n}^{(D)} \cdot \mathbf{n}^{(D)}=1$. The arbitrary initial state of the measured system $|\psi\rangle=a_{s}|0\rangle_{s}+b_{s}|1\rangle_{s}$ evolves under the system-detector coupling according to

$$
\begin{aligned}
|\psi\rangle\left|D_{i}\right\rangle & \rightarrow \exp \left[i \frac{g}{2}\left(1-\left(\mathbf{n}^{(s)} \cdot \boldsymbol{\sigma}^{(s)}\right)\right)\left(\mathbf{n}^{(D)} \cdot \boldsymbol{\sigma}^{(D)}\right)\right]|\psi\rangle\left|D_{i}\right\rangle \\
& =\left|\psi^{(0)}\right\rangle|0\rangle_{D}+\left|\psi^{(1)}\right\rangle|1\rangle_{D}
\end{aligned}
$$

where $g=\int_{0}^{T} d t \lambda(t)$. After the interaction has been switched off, $\sigma_{z}^{(D)}$ is measured projectively, yielding a readout $r \in$ $\{0,1\}$ corresponding to the postmeasurement detector states $|r\rangle_{D}$. The backaction matrices (representing the Kraus operators) are thus

$$
\mathcal{M}^{(r)}=R^{-1}\left(\mathbf{n}^{(s)}\right) M^{(r)} R\left(\mathbf{n}^{(s)}\right),
$$

with

$$
\begin{aligned}
M^{(0)} & =\left(\begin{array}{cc}
1 & 0 \\
0 & \cos g+i \sin g \cos \theta^{(D)}
\end{array}\right), \\
M^{(1)} & =\left(\begin{array}{cc}
0 & 0 \\
0 & i \sin g \sin \theta^{(D)} e^{i \varphi^{(D)}}
\end{array}\right), \\
R\left(\mathbf{n}^{(s)}\right) & =\left(\begin{array}{cc}
\cos \frac{\theta^{(s)}}{2} & \sin \frac{\theta^{(s)}}{2} e^{-i \varphi^{(s)}} \\
\sin \frac{\theta^{(s)}}{2} & -\cos \frac{\theta^{(s)}}{2} e^{-i \varphi^{(s)}}
\end{array}\right) .
\end{aligned}
$$

When $\mathbf{n}^{(s)}=(0,0,1)$, the matrices $M^{(r)}$ alone determine the backaction. For a general $\mathbf{n}^{(s)}$, the matrix $R\left(\mathbf{n}^{(s)}\right)$ induces a unitary rotation: The eigenbasis of $\left(\mathbf{n}^{(s)} \cdot \boldsymbol{\sigma}^{(s)}\right)=$ $R^{-1}\left(\mathbf{n}^{(s)}\right) \sigma_{z}^{(s)} R\left(\mathbf{n}^{(s)}\right)$ is given by $R^{-1}\left(\mathbf{n}^{(s)}\right)|0 / 1\rangle_{s}$. One thus sees that the role of $M^{(r)}$ is to determine the backaction in the eigenbasis of the measured observable $\left(\mathbf{n}^{(s)} \cdot \boldsymbol{\sigma}^{(s)}\right)$.

It is important to understand in detail the evolution of the system state during the measurement process. Consider the case of $\mathbf{n}^{(s)}=(0,0,1) .^{5}$ If the initial state $|\psi\rangle=|0\rangle_{s}$, the measurement yields $r=0$ with probability 1 and the state remains unchanged. For the initial state $|\psi\rangle=|1\rangle_{s}$, the probabilities of the readouts are $p_{r=0}=1-\sin ^{2} g \sin ^{2} \theta^{(D)}$ and $p_{r=1}=\sin ^{2} g \sin ^{2} \theta^{(D)}$; the state becomes $\left|\psi^{(r)}\right\rangle=e^{i \phi_{r}}|1\rangle_{s}$ with a readout-dependent phase $\phi_{r}$. For a generic initial state, both readouts are possible with some probabilities $p_{r}$ [cf. Eq. (4)], yet the backaction on the state does not reduce to a phase multiplication. The $r=1$ readout, whose backaction is described by $M^{(1)}$, projects the state onto $|1\rangle_{s}$. For $r=0$ readout, $M^{(0)}$ describes pulling the state towards the north pole on the Bloch sphere (i.e., closer to $|0\rangle_{s}$ ) and rotating it around the $z$ axis (cf. Fig. 1).

This rotation is a key feature of weak measurement and is absent in the case of projective measurements. Indeed, in a projective measurement, the $r=0$ readout would imply the final state $|0\rangle_{s}$ and any rotation around the $z$ axis would become insignificant. Note that this rotation only happens when $M^{(0)}$ has an imaginary component, i.e., when $\mathcal{M}^{(0)}$ is non-Hermitian. The idiosyncrasy of Hermitian backaction matrices has been discussed in Sec. IIC. It is this non-Hermitian backaction that gives rise to asymmetric state trajectories as shown in Fig. 2 (see Fig. 3 for a detailed explanation).

\footnotetext{
${ }^{5}$ For arbitrary $\mathbf{n}^{(s)}$, the effect is the same if considered in the eigenbasis of $\left(\mathbf{n}^{(s)} \cdot \boldsymbol{\sigma}^{(s)}\right)$.
} 


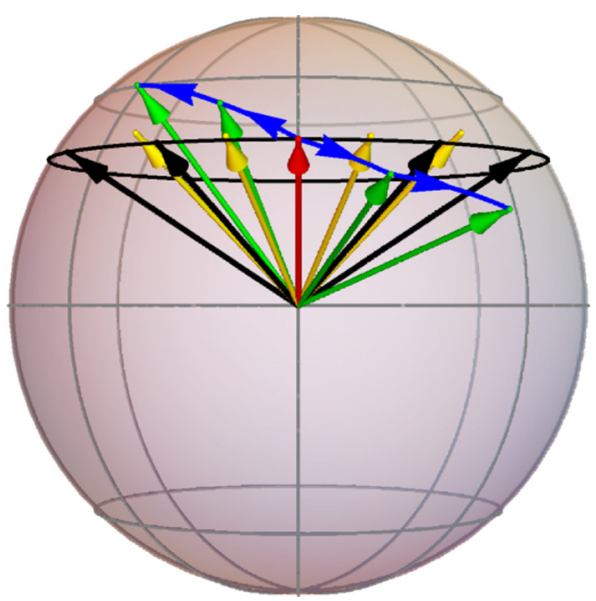

FIG. 3. Emergence of asymmetry of time-reversed measurement-induced trajectories. Two measurement sequences of spin projections $\left(\mathbf{n}^{(s)} \cdot \boldsymbol{\sigma}^{(s)}\right)$ onto subsequent directions $\mathbf{n}^{(s)}$ located on the same parallel (black line) are performed, a clockwise and a counterclockwise one, respectively. We illustrate the effect of the first two measurements of each sequence (clockwise and counterclockwise). All measurements are assumed to yield readouts $r=0$ and are characterized by Kraus operators $\mathcal{M}^{(0)}$ [Eq. (28)]. The initial state is represented by the red arrow and the measurement axes are shown by black arrows. If the backaction operator $\mathcal{M}^{(0)}$ is Hermitian, the clockwise and the counterclockwise trajectories are mirror reflections of each other (yellow arrows). However, for non-Hermitian backaction, there is an extra rotation around the measurement axes (cf. Fig. 1) leading to the two trajectories not being simply related to each other (green arrows show the state locations after the respective measurements; these locations are connected by the blue arrows to show what the clockwise trajectory is and what the counterclockwise one is).

We note in passing that the backaction matrices in Eqs. (29) and (30) can appear in a wider context than the toy detector model introduced here. In some contexts, measurements with such backaction are known as partial or interaction-free measurements [33-35] and can be implemented by optical means [33,35] or in superconducting qubits [34]. A particularly natural setting for such backaction is presented by imperfect optical polarizers. A polarizer is a detector in the sense that the light can pass through the polarizer (identified with $r=0$ ) or not pass (be absorbed, reflected, etc., with $r=1$ ). An ideal polarizer, letting one polarization through and completely reflecting the other, is equivalent to a strong measurement being applied to a photon. A nonideal polarizer, letting one polarization through completely while the other polarization is partially reflected and partially transmitted, can be described as a measurement with backaction matrices of the form (29) and (30). Therefore, our proposed protocols, detailed below, may in principle be implemented in a variety of experimental settings.

\section{B. Measurement sequences and scaling limit}

Hereafter we focus on studying a specific family of measurement sequences. We consider the system initial state

$$
\left|\psi_{0}\right\rangle=\cos \frac{\theta}{2}|0\rangle_{s}+\sin \frac{\theta}{2}|1\rangle_{s} .
$$

We choose our measurements to be associated with the measurement axes

$$
\mathbf{n}_{k}^{(s)}=\left(\sin \theta_{k}^{(s)} \cos \varphi_{k}^{(s)}, \sin \theta_{k}^{(s)} \sin \varphi_{k}^{(s)}, \cos \theta_{k}^{(s)}\right),
$$

where

$$
\left(\theta_{k}, \varphi_{k}\right)=(\theta, 2 \pi k d /(N+1)),
$$

i.e., all the measurement axes belong to a particular parallel corresponding to the polar angle $\theta$, and $d= \pm 1$ denotes whether the sequence is performed clockwise/counterclockwise. We fix the measurement parameters $g_{k}$ and $\mathbf{n}_{k}^{(D)}$ to be $g$ and $\mathbf{n}^{(D)}=\left(\sin \theta^{(D)} \cos \varphi^{(D)}\right.$, $\left.\sin \theta^{(D)} \sin \varphi^{(D)}, \cos \theta^{(D)}\right)$, independently of the measurement number $k$. For simplicity, we set $\varphi^{(D)}=-\pi / 2$.

We will be interested in the limit $N \rightarrow \infty$, where the measurement sequence becomes quasicontinuous. If one keeps $g$ and $\theta^{(D)}$ constant when taking the $N \rightarrow \infty$ limit, a sequence of an infinite number of finite strength measurements becomes equivalent to a sequence of projective measurements and yields the Pancharatnam phase (cf. Appendix A). In order to avoid this trivial limiting case, one needs to scale $g$ and $\theta^{(D)}$ with $N$. In Appendix A we show that among the large number of possible approaches to the continuum limit there is a unique scaling procedure that avoids a trivial limit.

This nontrivial scaling procedure corresponds to $g=$ $\sqrt{4 C / N}$ and $\theta^{(D)}=\pi / 2+A / \sqrt{C N}$ with the parameters $C \geqslant$ 0 and $A \in \mathbb{R}$. With such scaling, the backaction matrices in Eqs. (29) and (30) become

$$
\begin{gathered}
M^{(0)}=\left(\begin{array}{cc}
1 & 0 \\
0 & \exp \left(-2 \frac{C+i A}{N}\right)+O\left(\frac{1}{N^{2}}\right)
\end{array}\right), \\
M^{(1)}=\left(\begin{array}{lc}
0 & 0 \\
0 & \sqrt{\frac{4 C}{N}}+O\left(\frac{1}{N^{3 / 2}}\right)
\end{array}\right) .
\end{gathered}
$$

The parameter $C$ controls the measurement strength (how much the state is pulled towards the measurement axis for the $r=0$ readout), while $A \in \mathbb{R}$ controls the non-Hermiticity of $M^{(0)}$ [and $\mathcal{M}^{(0)}$ in Eq. (28)]. Since non-Hermiticity is the cause of asymmetric behavior (as was shown in Secs. II C and III A), we call $A$ the asymmetry parameter.

The non-Hermitian contribution to the measurement backaction can be interpreted as Hamiltonian evolution

$$
\begin{aligned}
M^{(0)} & =\left(\begin{array}{cc}
1 & 0 \\
0 & \exp \left(-2 \frac{C+i A}{N}\right)
\end{array}\right) \\
& =\left(\begin{array}{cc}
1 & 0 \\
0 & \exp \left(-2 \frac{C}{N}\right)
\end{array}\right) \exp (-i H \Delta t),
\end{aligned}
$$

where $\Delta t=N^{-1}$ and $H=A\left(\mathbb{I}-\sigma_{z}^{(s)}\right)$. Therefore, this backaction could in principle arise as a result of a measurement with Hermitian backaction applied to a system evolving under the Hamiltonian $H$. This, however, is not how the backaction emerges here: The system does not have its own Hamiltonian, nor does the detector model have any term in the Hamiltonian (25) acting solely on the system. Nevertheless, Eq. (37) shows that for the purposes of investigating the effect on the system state, the measurements we consider are equivalent to measurements with a Hermitian backaction (determined by $C$ ) supplemented with Hamiltonian evolution of the system (determined by $A$ ). We find this equivalence useful for connecting 
our results to the known results for Hamiltonian-evolutioninduced phase factors in Sec. III E $3{ }^{6}$

\section{Measurement-induced phase in postselected measurement sequences}

Here we investigate the behavior of the postselected phase $\chi_{\left\{r_{k}\right\}}^{(d= \pm 1)}$ defined in Sec. II, Eqs. (19) and (20). We focus on a specific readout sequence in which all detector readouts are $r_{k}=0$. Such a choice is based on the following observation. Within the measurement model described in Sec. III A, $r=0$ readout implies that the detector state before a measurement coincides with the detector state after the measurement. This allows for designing a simple observation scheme for $\chi_{\left\{r_{k}=0\right\}}^{(d)}$, as described in Sec. VI.
The parameter $d= \pm 1$ denotes the direction of the measurement sequence [cf. Eq. (34)]. We next calculate $\chi_{\left\{r_{k}=0\right\}}^{(d)}$ for both directions, keeping $d$ unspecified. Using Eqs. (28)-(31) and the explicit definitions for the initial state $\left|\psi_{0}\right\rangle$ [Eq. (32)], the measurement axes $\mathbf{n}_{k}^{(s)}$ [Eq. (33)], and the protocol direction $d$ [Eq. (34)], we show that

$$
\begin{aligned}
& \sqrt{P_{\left\{r_{k}=0\right\}}^{(d)}} e^{i \chi_{\left\{r_{k}=0\right\}}^{(d)}}=\left\langle\psi_{0}\left|\mathcal{M}_{N}^{(0)} \cdots \mathcal{M}_{1}^{(0)}\right| \psi_{0}\right\rangle \\
& =\left(\begin{array}{ll}
1 & 0
\end{array}\right) \delta R^{(d)}\left(M^{(0)} \delta R^{(d)}\right)^{N}\left(\begin{array}{l}
1 \\
0
\end{array}\right),
\end{aligned}
$$

where

$$
\delta R^{(d)}=R\left(\mathbf{n}_{k}^{(s)}\right) R^{-1}\left(\mathbf{n}_{k-1}^{(s)}\right)=\left(\begin{array}{cc}
\cos ^{2} \frac{\theta}{2}+\sin ^{2} \frac{\theta}{2} \exp \left(-\frac{2 \pi i d}{N+1}\right) & \frac{1}{2}\left[1-\exp \left(-\frac{2 \pi i d}{N+1}\right)\right] \sin \theta \\
\frac{1}{2}\left[1-\exp \left(-\frac{2 \pi i d}{N+1}\right)\right] \sin \theta & \sin ^{2} \frac{\theta}{2}+\cos ^{2} \frac{\theta}{2} \exp \left(-\frac{2 \pi i d}{N+1}\right)
\end{array}\right)
$$

Using Eq. (35), diagonalizing $M^{(0)} \delta R^{(d)}$, and taking the limit of $N \rightarrow \infty$, one finds that

$$
\sqrt{P_{\left\{r_{k}=0\right\}}^{(d)}} e^{i \chi_{\left\{r_{k}=0\right\}}^{(d)}}=e^{i \pi d(\cos \theta-1)-Z}\left(\cosh \tau+Z \frac{\sinh \tau}{\tau}\right),
$$

where $Z=C+i A+i \pi d \cos \theta$ and $\tau=\sqrt{Z^{2}-\pi^{2} \sin ^{2} \theta}$. Note that the definition of $\tau$ through the square root allows for a sign ambiguity. Since Eq. (40) is symmetric under $\tau \rightarrow$ $-\tau$, the actual sign does not matter and one can choose any convention for calculating the square root. Note also that the prefactor $e^{i \pi d(\cos \theta-1)}$ is exactly the Pancharatnam phase of the system subjected to a quasicontinuous sequence of projective measurements along the parallel corresponding to $\theta$.

The right-hand side of Eq. (40) obeys a number of symmetries. First, the expression is invariant under simultaneous replacement of $d \rightarrow-d$ and $\theta \rightarrow \pi-\theta$. Second, the expression remains unaffected under $d \rightarrow-d$ and $A \rightarrow-A$ accompanied by the complex conjugation. From the latter, it follows that for $A=0, P_{\left\{r_{k}=0\right\}}^{(d=+1)}=P_{\left\{r_{k}=0\right\}}^{(d=-1)}$ and $\chi_{\left\{r_{k}=0\right\}}^{(d=+1)}=$ $-\chi_{\left\{r_{k}=0\right\}}^{(d=-1)}(\bmod 2 \pi)$. That is, at $A=0$ the probability only has a nontrivial symmetric component and the phase only has the antisymmetric component. Away from $A=0$, the phase

\footnotetext{
${ }^{6}$ Note also that the scaling of the backaction matrix in Eq. (35) is the "natural" one in the following case: The measurements are implemented with polarizers, where the degree of polarization is determined by the polarizer thickness. Indeed, for such a polarizer, the degree of letting the "wrong" polarization through would drop exponentially with the thickness $L$ of the polarizer. At the same time, different refraction indices for the two polarizations would also result in a phase difference proportional to $L$. Adjusting the polarizer thickness $L \sim N^{-1}$ according to the number $N$ of measurements employed would result in the backaction given in Eq. (35), applied to the polarization of the transmitted light. This should enable a relatively easy check of our predictions concerning the case when all the measurements are postselected to yield $r_{k}=0$ (cf. Secs. III C, IV, and VI A).
}

and the postselection probability have both symmetric and antisymmetric components (17), (22), and (23).

\section{How to calculate the averaged phase}

Here we derive a relatively simple expression for the averaged measurement-induced phase $\bar{\chi}^{(d)}$ in Eq. (21). While our result does not constitute a fully analytical expression for $\bar{\chi}^{(d)}$, it facilitates general analysis and efficient numerical study of the averaged phase behavior.

Note that, similarly to Eq. (38), for an arbitrary readout sequence $\left\{r_{k}\right\}$,

$$
\begin{aligned}
& \left\langle\psi_{0}\left|\mathcal{M}_{N}^{\left(r_{N}\right)} \cdots \mathcal{M}_{1}^{\left(r_{1}\right)}\right| \psi_{0}\right\rangle
\end{aligned}
$$

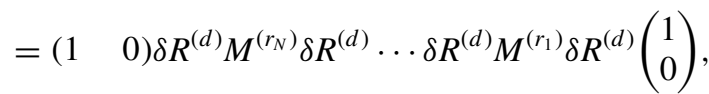

with $M^{\left(r_{k}\right)}$ defined in Eqs. (35) and (36) and $\delta R^{(d)}$ defined in Eq. (39). Then

$$
\begin{aligned}
& e^{2 i \bar{\chi}^{(d)}-\alpha^{(d)}} \\
& \quad=\sum_{\left\{r_{k}\right\}}\left(\left\langle\psi_{0}\left|\mathcal{M}_{N}^{\left(r_{N}\right)} \cdots \mathcal{M}_{1}^{\left(r_{1}\right)}\right| \psi_{0}\right\rangle\right)^{2} \\
& \quad=\sum_{\left\{r_{k}\right\}}\left(\begin{array}{l}
1 \\
0
\end{array}\right)^{T} \otimes\left(\begin{array}{l}
1 \\
0
\end{array}\right)^{T} \delta R_{4}^{(d)} M_{4}^{\left(r_{N}\right)} \cdots M_{4}^{\left(r_{1}\right)} \delta R_{4}^{(d)}\left(\begin{array}{l}
1 \\
0
\end{array}\right) \otimes\left(\begin{array}{l}
1 \\
0
\end{array}\right) \\
& =\left(\begin{array}{l}
1 \\
0
\end{array}\right)^{T} \otimes\left(\begin{array}{l}
1 \\
0
\end{array}\right)^{T} \delta R_{4}\left(\mathfrak{M}^{(d)}\right)^{N}\left(\begin{array}{l}
1 \\
0
\end{array}\right) \otimes\left(\begin{array}{l}
1 \\
0
\end{array}\right),
\end{aligned}
$$

where $\delta R_{4}^{(d)}=\delta R^{(d)} \otimes \delta R^{(d)}, M_{4}^{\left(r_{k}\right)}=M^{\left(r_{k}\right)} \otimes M^{\left(r_{k}\right)}$, $\otimes$ denotes the tensor product, and $\mathfrak{M}^{(d)}=\sum_{r} M_{4}^{(r)} \delta R_{4}^{(d)}$. Therefore,

$$
\begin{aligned}
& e^{2 i \bar{\chi}^{(d)}-\alpha^{(d)}}=\left(\begin{array}{llll}
1 & 0 & 0 & 0
\end{array}\right) \delta R_{4}^{(d)}\left(\mathfrak{M}^{(d)}\right)^{N}\left(\begin{array}{lllll}
1 & 0 & 0 & 0
\end{array}\right)^{T} \\
& =\left[\delta R_{4}^{(d)}\left(\mathfrak{M}^{(d)}\right)^{N}\right]_{11},
\end{aligned}
$$


where

$$
\mathfrak{M}^{(d)}=\left(\begin{array}{cccc}
1+\frac{2 i \pi d \cos \theta}{N} & -\frac{i \pi d \sin \theta}{N} & -\frac{i \pi d \sin \theta}{N} & 0 \\
-\frac{i \pi d \sin \theta}{N} & 1-2 \frac{C+i A}{N} & 0 & -\frac{i \pi d \sin \theta}{N} \\
-\frac{i \pi d \sin \theta}{N} & 0 & 1-2 \frac{C+i A}{N} & -\frac{i \pi d \sin \theta}{N} \\
0 & -\frac{i \pi d \sin \theta}{N} & -\frac{i \pi d \sin \theta}{N} & 1-\frac{2 i \pi d \cos \theta}{N}-\frac{4 i A}{N}
\end{array}\right)+O\left(\frac{1}{N^{2}}\right) .
$$

What enabled a fully analytical calculation in Sec. IIIC is the possibility to diagonalize $M^{(0)} \delta R^{(d)}$ analytically. Here, diagonalizing $\mathfrak{M}^{(d)}$ analytically is a formidable task. However, it can be diagonalized numerically. Suppose one diagonalized $\mathfrak{M}^{(d)}$,

$$
\mathfrak{M}^{(d)}=V D V^{-1},
$$

with $D=\operatorname{diag}\left(\lambda_{1}, \lambda_{2}, \lambda_{3}, \lambda_{4}\right)$ and $\lambda_{j}=1+x_{j} / N+O\left(N^{-2}\right)$. Then in the limit $N \rightarrow \infty$,

$$
e^{2 i \bar{\chi}^{(d)}-\alpha^{(d)}}=\left[\left(\mathfrak{M}^{(d)}\right)^{N}\right]_{11}=\left[V \mathcal{D} V^{-1}\right]_{11},
$$

with $\mathcal{D}=\operatorname{diag}\left(e^{x_{1}}, e^{x_{2}}, e^{x_{3}}, e^{x_{4}}\right)$.

The expressions (44) and (46) not only provide a means for efficient numeric calculation of the averaged phase, they also allow one to make some analytic conclusions. Namely, one can show that the averaged phase obeys the same symmetries as the postselected phase (cf. Sec. IIIC). Observe that $\left.\mathfrak{M}^{(d)}\right|_{\theta \rightarrow \pi-\theta}=U^{-1} \mathfrak{M}^{(-d)} U$, where $U=\operatorname{diag}(1,-1,-1,1)$. Therefore, $e^{2 i \bar{\chi}^{(d)}-\alpha^{(d)}}$ remains invariant under simultaneous replacement of $d \rightarrow-d$ and $\theta \rightarrow \pi-\theta$ :

$$
\begin{aligned}
\left.e^{2 i \overline{\bar{\chi}}(-d)-\alpha^{(-d)}}\right|_{\theta \rightarrow \pi-\theta} & =\lim _{N \rightarrow \infty}\left[\left(\left.\mathfrak{M}^{(-d)}\right|_{\theta \rightarrow \pi-\theta}\right)^{N}\right]_{11} \\
& =\lim _{N \rightarrow \infty}\left[\left(U^{-1} \mathfrak{M}^{(d)} U\right)^{N}\right]_{11}=\lim _{N \rightarrow \infty}\left[U^{-1}\left(\mathfrak{M}^{(d)}\right)^{N} U\right]_{11} \\
& =\lim _{N \rightarrow \infty}\left[\left(\mathfrak{M}^{(d)}\right)^{N}\right]_{11}=e^{2 i \bar{\chi}^{(d)}-\alpha^{(d)}} .
\end{aligned}
$$

Further, $\left.\mathfrak{M}^{(-d)}\right|_{A \rightarrow-A}=\left(\mathfrak{M}^{(d)}\right)^{*}$, implying that $e^{2 i \bar{\chi}^{(d)}-\alpha^{(d)}}$ is invariant under applying complex conjugation and simultaneously replacing $d \rightarrow-d$ and $A \rightarrow-A$.

\section{E. Limiting cases}

The analytic results of Secs. III C and III D allow one to analyze the behavior of the postselected [Eq. (40)] and averaged [Eq. (46)] phases in a number of limiting cases. In this section we discuss three limiting cases corresponding to $A \rightarrow \infty$, $C \rightarrow \infty$, and $C=0$.

\section{Case of $A \rightarrow \infty$}

We start with the simplest limiting case of $A \rightarrow \infty$. This means that the backaction of $r=0$ readouts strongly rotates the system state around the measurement axis [cf. Eq. (35)]. This regime is equivalent to an almost adiabatic Hamiltonian evolution supplemented by measurements that have a small effect [cf. Eq. (37) in Sec. III B]. Consequently, one expects the measurementinduced phase in this limit to coincide with the Berry phase $\pi d(\cos \theta-1)$ up to small corrections. This indeed turns out to be the case. For the postselective protocol, we expand the logarithm of Eq. (40) at large A. For the averaging protocol, we perform the diagonalization of Eq. (44) approximately at $A \rightarrow \infty$, after which we use Eq. (46). In both cases, we obtain

$$
\begin{gathered}
\chi_{\left\{r_{k}=0\right\}}^{(d)}=\bar{\chi}^{(d)}=\pi d(\cos \theta-1)+\frac{\pi^{2} \sin ^{2} \theta}{2 A}-\frac{\pi^{2} \sin ^{2} \theta}{4 A^{2}}\left[e^{-2 C} \sin (2 A+2 \pi d \cos \theta)-2 \pi d \cos \theta\right]+O\left(A^{-3}\right), \\
P_{\left\{r_{k}=0\right\}}^{(d)}=e^{-\alpha^{(d)}}=\exp \left(-\frac{\pi^{2} \sin ^{2} \theta}{2 A^{2}}\left[1+2 C-e^{-2 C} \cos (2 A+2 \pi d \cos \theta)\right]+O\left(A^{-3}\right)\right) .
\end{gathered}
$$

It is noteworthy that the results for the postselective and for the averaging protocols coincide as the $r_{k} \neq 0$ readouts have negligible probability. At higher orders in $A^{-1}$, this is no longer so.

Note that the phases $\chi_{\left\{r_{k}=0\right\}}^{(d)}$ and $\bar{\chi}^{(d)}$ do not possess a definite symmetry under $d \rightarrow-d$. In other words, they feature both symmetric and antisymmetric components, in agreement with the symmetry-based analysis in Secs. IIIC and IIID. The same applies to the postselection probability $P_{\left\{r_{k}=0\right\}}^{(d)}$ and the dephasing factor $e^{-\alpha^{(d)}}$.

\section{Case of $C \rightarrow \infty$}

The limit of $C \rightarrow \infty$ corresponds to almost projective measurements. Here one expects the induced phase to be the Pancharatnam phase $\pi d(\cos \theta-1)$ up to small corrections. For the postselective protocol, expanding Eq. (40), we find

$$
\chi_{\left\{r_{k}=0\right\}}^{(d)}=\pi d(\cos \theta-1)+\frac{\pi^{2} \sin ^{2} \theta}{2 C^{2}}[A+\pi d \cos \theta]+O\left(C^{-3}\right),
$$




$$
P_{\left\{r_{k}=0\right\}}^{(d)}=\exp \left(-\frac{\pi^{2} \sin ^{2} \theta}{C}\left[1-\frac{1}{2 C}\right]+O\left(C^{-3}\right)\right) .
$$

For the averaging protocol, we find

$$
\begin{gathered}
\bar{\chi}^{(d)}=\pi d(\cos \theta-1)+\frac{\pi^{2} \sin ^{2} \theta}{2 C^{2}}\left[A+\pi d \cos \theta-\pi^{2} \sin ^{2} \theta \frac{\sin (4 A+4 \pi d \cos \theta)-4(A+\pi d \cos \theta)}{16(A+\pi d \cos \theta)^{2}}\right]+O\left(C^{-3}\right), \\
e^{-\alpha^{(d)}}=\exp \left(-\frac{\pi^{2} \sin ^{2} \theta}{C}\left[1-\frac{1}{2 C}\right]+\frac{\pi^{4} \sin ^{4} \theta}{2 C^{2}}\left[\frac{\sin (2 A+2 \pi d \cos \theta)}{2(A+\pi d \cos \theta)}\right]^{2}+O\left(C^{-3}\right)\right) .
\end{gathered}
$$

Note that the asymmetry with respect to $d \rightarrow-d$ is present in the postselected phase but not in the postselection probability (where it only appears in terms proportional to $C^{-3}$ ). At the same time, the asymmetry does appear in $\alpha^{(d)}$ at this order, showing the nontrivial effect of averaging.

When $C \rightarrow \infty$, one recovers the limit of projective measurements, implying that the resulting phase $\chi_{\left\{r_{k}=0\right\}}^{(d)}$ is the Pancharatnam phase. Since the postselection probability $P_{\left\{r_{k}=0\right\}}^{(d)}=1$, other readout sequences cannot occur, and the averaged phase is the same as the postselected one. At large but finite values of $C$, the two phases are different. One can clearly see the separation of the $\left\{r_{k}=0\right\}$ contribution from that of all the other readout sequences in both the phases and the postselection probability/dephasing factor. Remarkably, the other sequences contribute only at $O\left(C^{-2}\right)$.

\section{Case of $C=0$}

This limit corresponds to zero strength measurement. The measurements always yield $r=0$ readouts, and the corresponding backaction (35) is equivalent to a Hamiltonian evolution [cf. Eq. (37)]. On one hand, this can still be interpreted as the behavior under very weak measurements. On the other hand, this limit can be understood as nonadiabatic Hamiltonian evolution and treated within the framework of Aharonov-Anandan phases [11]. As we show below, the two treatments yield identical results.

The answer in this limit immediately follows from Eq. (40), which yields

$$
\sqrt{P_{\left\{r_{k}=0\right\}}^{(d)}} e^{i \chi_{\left.l_{k}=0\right\}}^{(d)}}=e^{2 i \bar{\chi}^{(d)}-\alpha^{(d)}}=-e^{-i A}\left(\cos \zeta+Z \frac{\sin \zeta}{\zeta}\right),
$$

where $\quad Z=i A+i \pi d \cos \theta \quad$ and $\quad \zeta=$ $\sqrt{(A+\pi d \cos \theta)^{2}+\pi^{2} \sin ^{2} \theta}$. Using the same technique as in Sec. III C, it is possible to obtain the analytic form of the geometrical component of the phase (14):

$$
\begin{aligned}
\arg \left\langle\psi_{0}\left|\mathcal{P}_{N} \cdots \mathcal{P}_{1}\right| \psi_{0}\right\rangle= & \arg \left[-e^{-i A}\left(\cos \zeta+Z \frac{\sin \zeta}{\zeta}\right)\right. \\
& \left.\times \exp \left(i \frac{A \pi^{2} \sin ^{2} \theta}{\zeta^{2}}\left\{1-\frac{\sin 2 \zeta}{2 \zeta}\right\}\right)\right] .
\end{aligned}
$$

The dynamical part of the phase is thus

$$
\chi_{\left\{r_{k}=0\right\}}^{(d)}-\arg \left\langle\psi_{0}\left|\mathcal{P}_{N} \cdots \mathcal{P}_{1}\right| \psi_{0}\right\rangle=-\frac{A \pi^{2} \sin ^{2} \theta}{\zeta^{2}}\left\{1-\frac{\sin 2 \zeta}{2 \zeta}\right\} .
$$

One sees that neither the dynamical nor the geometrical part of the phase possesses a definite symmetry under $d \rightarrow-d$. Each has both a symmetric and an antisymmetric component.

In the present case $C=0$, separation into the dynamical and geometrical components can be obtained following Aharonov and Anandan [11]. Indeed, the measurement backaction can be interpreted as Hamiltonian evolution

$$
\begin{aligned}
& \mathcal{M}_{k}^{(0)}=\exp \left(-i H_{k} \Delta t\right), \quad \mathcal{M}_{k}^{(1)}=0 ; \\
& H_{k}=A\left(\mathbb{I}-\mathbf{n}_{k}^{(s)} \cdot \sigma^{(s)}\right), \quad \Delta t=N^{-1}
\end{aligned}
$$

[cf. Eqs. (28)-(31) and (35)-(37)]. Then the dynamical phase $-\sum_{k=0}^{N-1}\left\langle\psi_{k}\left|H_{k+1}\right| \psi_{k}\right\rangle \Delta t$ in the limit $N \rightarrow \infty$ is given exactly by the right-hand side of Eq. (56). This demonstrates consistency between our definition of the geometrical and dynamical components of measurement-induced phases and the conventional definition for the phases induced by Hamiltonian evolution. Further investigation of the separation of the measurement-induced phases into dynamical and geometrical components is left for future work.

\section{TOPOLOGICAL TRANSITIONS IN THE POSTSELECTIVE PROTOCOL}

In this section we investigate topological transitions concerning the postselected phase $\chi_{\left\{r_{k}=0\right\}}^{(d)}$. We study these transitions in Sec. IV A and discuss the resulting phase diagram in the space of measurement parameters in Sec. IV B.

\section{A. Essence of transitions}

Consider the postselection probability $P_{\left\{r_{k}=0\right\}}^{(d)}$ [cf. Eqs. (19), (20), and (40)] when the protocol is executed at, for example, $\theta=3 \pi / 4$ [cf. Fig. 4(a)]. We note that $\ln P_{\left\{r_{k}=0\right\}}^{(d=+1)}(\theta=3 \pi / 4)$ is bounded throughout the entire parameter space except for a divergence near $(C=1, A=2)$, indicating that $P_{\left\{r_{k}=0\right\}}^{(=+1)} \rightarrow$ 0 at this special point. This is accompanied by a prominent feature in the behavior of $\chi_{\left\{r_{k}=0\right\}}^{(d=+1)}(\theta=3 \pi / 4)$ [cf. Fig. 4(b)]: The phase $\chi_{\left\{r_{k}=0\right\}}^{(d=+1)}(\theta=3 \pi / 4)$ is ill-defined at the singularity and makes a $2 \pi$ winding around the singular point. This is a topological feature in the sense that it cannot be eliminated by a smooth deformation of $\chi_{\left\{r_{k}=0\right\}}^{(d=+1)}(\theta=3 \pi / 4)$ as a function 

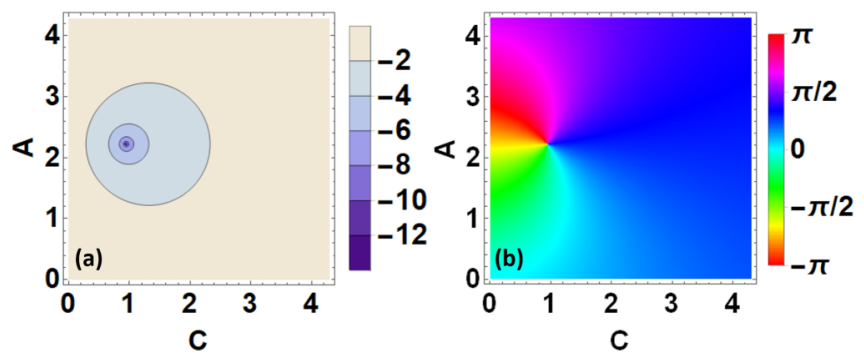

FIG. 4. Vanishing of the probability and windings of the phase in the postselective protocol $\left\{r_{k}=0\right\}$. (a) Contour plot of the logarithm of the postselection probability $\ln P_{\left\{r_{k}=0\right\}}^{(d=+1)}(\theta=3 \pi / 4)$ (value indicated by color) as a function of measurement parameters $C$ and $A$ [cf. Eq. (40)]. Note the divergence at $(C, A) \approx(1,2)$. (b) Dependence of the phase $\chi_{\left\{r_{k}=0\right\}}^{(d=+1)}(\theta=3 \pi / 4)$ (value indicated by color) on $C$ and $A$. The phase is ill-defined at the singularity point $(C, A) \approx(1,2)$. Following the phase value around the singular point, the phase varies continuously from $-\pi$ to $\pi$, i.e., makes a $2 \pi$ winding.

of $(C, A)$. Similar features emerge at other values of $\theta$, with different locations of the special point in the $(C, A)$ plane.

For an arbitrary $\theta$, the presence of a phase winding implies that the phase is ill-defined at a certain value of $(C, A)$. In Sec. VIA we show that $\sqrt{P_{\left\{r_{k}=0\right\}}^{(d)}} e^{i \chi_{\left\{r_{k}=0\right\}}^{(d)}}$ is an observable quantity. Therefore, the phase $\chi_{\left\{r_{k}=0\right\}}^{(d)}(\theta)$ being illdefined at $\left(C_{\text {crit }}, A_{\text {crit }}\right)$ implies $P_{\left\{r_{k}=0\right\}}^{(d)}\left(\theta, C_{\text {crit }}, A_{\text {crit }}\right)=0$. The converse is not necessarily true. However, in our study of measurement-induced phases we have not found instances of the postselection probability vanishing without a phase singularity.

In Sec. IV B we find the set of all points $\left(C_{\text {crit }}, A_{\text {crit }}, \theta_{\text {crit }}\right)$ corresponding to the postselection probability vanishing. Before proceeding there, we now present a different view of what happens at these special points.

By construction [cf. Eqs. (19) and (20)], for each given $\theta$, $\chi_{\left\{r_{k}=0\right\}}^{(d)}(\theta)$ is defined modulo $2 \pi$. It follows from Eq. (40) that $\chi_{\left\{r_{k}=0\right\}}^{(d)}(\theta=0)=\chi_{\left\{r_{k}=0\right\}}^{(d)}(\theta=\pi)=0(\bmod 2 \pi)$. Without loss of generality, one can assign $\chi_{\left\{r_{k}=0\right\}}^{(d)}(\theta=0)=0$. On top of that, demanding the continuity of $\chi_{\left\{r_{k}=0\right\}}^{(\theta)}(\theta)$ as a function of $\theta$, one removes the freedom of adding multiples of $2 \pi$ to $\chi_{\left\{r_{k}=0\right\}}^{(d)}(\theta)$. One thus must have $\chi_{\left\{r_{k}=0\right\}}^{(d)}(\theta=\pi)=2 \pi n$, where $n$ is a well-defined integer that characterizes the entire dependence of $\chi_{\left\{r_{k}=0\right\}}^{(d)}$ on $\theta$ at a given $(C, A)$. It is natural to denote by $n$ the winding number, as it represents the number of times the function $e^{i \chi_{\left.l_{k}=0\right\}}^{(d)}(\theta)}$ winds around the origin in the complex plane. Being an integer number,

$$
n=\frac{1}{2 \pi} \int_{0}^{\pi} d \theta \frac{d \chi_{\left\{r_{k}=0\right\}}^{(d)}(\theta)}{d \theta}=\frac{\chi_{\left\{r_{k}=0\right\}}^{(d)}(\pi)-\chi_{\left\{r_{k}=0\right\}}^{(d)}(0)}{2 \pi}
$$

cannot change as $\chi_{\left\{r_{k}=0\right\}}^{(d)}(\theta)$ is smoothly deformed, rendering $n$ a topological invariant. The presence of different values of $n$ at different measurement parameters $C$ and $A$ implies the existence of a sharp transition where the value of $n$ jumps discontinuously. In other words, there must exist some critical $\left(C_{\text {crit }}, A_{\text {crit }}\right)$ at which the function $\chi_{\left\{r_{k}=0\right\}}^{(d)}(\theta)$ is ill-defined; it

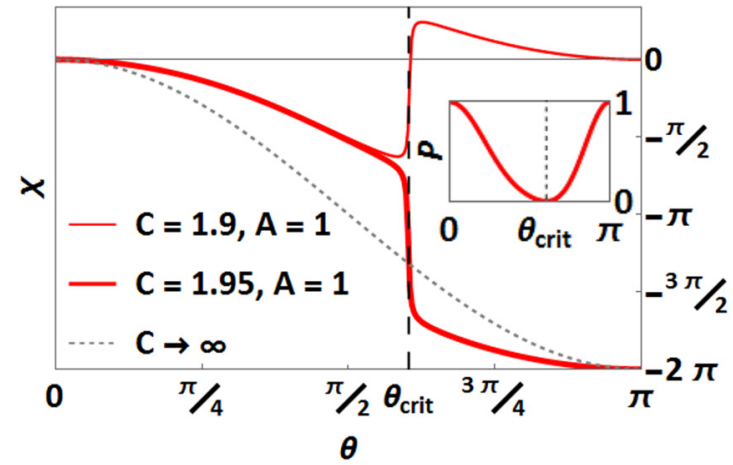

FIG. 5. Phase $\chi_{\left\{r_{k}=0\right\}}^{(d=+1)} \equiv \chi$ [cf. Eq. (40)] as a function of $\theta$ for $A=1$ for $C$ above and below the critical value $C_{\text {crit }} \approx 1.925$. The winding number $n$ [cf. Eq. (59)] is equal to 0 for $C<C_{\text {crit }}$ and to -1 for $C>C_{\text {crit }}$. The behavior of $\chi\left(\theta<\theta_{\text {crit }}\right)$ immediately above and below the transition is identical, while the dependence of $\chi\left(\theta>\theta_{\text {crit }}\right)$ differs by a $2 \pi$ shift. This leads to $\chi\left(\theta=\theta_{\text {crit }}, C=C_{\text {crit }}\right)$ being illdefined. The dependence of $P_{\left\{r_{k}=0\right\}}^{(d=+1)} \equiv P$ on $\theta$ at $\left(C=C_{\text {crit }}, A=1\right)$ is shown in the inset. The indeterminacy of $\chi\left(\theta=\theta_{\text {crit }}, C=C_{\text {crit }}\right)$ is enabled by $P\left(\theta=\theta_{\text {crit }}, C=C_{\text {crit }}\right)=0$.

is sufficient for $\chi_{\left\{r_{k}=0\right\}}^{(d)}(\theta)$ not to be well defined at a single $\theta=\theta_{\text {crit }}$. As discussed above, this requires $P_{\left\{r_{k}=0\right\}}^{(d)}\left(\theta_{\text {crit }}\right)=0$. Hence, such transitions between different values of the winding number $n$ correspond to singularities like the one found above.

Such transitions were reported in Ref. [20] for the case of $A=0$. There the existence of such transitions is evident through a simple consideration. For the limit of infinitely weak measurements $(C=A=0), \chi_{\left\{r_{k}=0\right\}}^{(d)}(\theta) \equiv 0$, yielding $n=0$, while in the limit of projective measurements $[(C \rightarrow$ $\infty, A=0)], \chi_{\left\{r_{k}=0\right\}}^{(d)}(\theta)=\pi d(\cos \theta-1)$, yielding $n=-d$. Therefore, there must be a transition at some finite $C>0$ when $A=0$.

For the present, more general case, the above consideration does not apply. While at $C \rightarrow \infty, \chi_{\left\{r_{k}=0\right\}}^{(d)}(\theta)=\pi d(\cos \theta-1)$ and $n=-d$ for any $A$ [cf. Eq. (40)], the phase at $(C=0, A \neq$ 0 ) is not identically zero. Therefore, one cannot guarantee the existence of a transition at a certain $C$ for an arbitrary value of $A$. We find that transitions exist for $|A| \leqslant A_{0}=\pi \sqrt{3} / 2$ (cf. Sec. IV B and Appendix B) and do not exist otherwise. An example of such a transition is presented in Fig. 5.

Reference [20] also linked this type of transition to a topological transition of the surface formed by measurementinduced trajectories on the Bloch sphere. Consider the sequence of states $\left\{\left|\psi_{k=0, \ldots, N}\right\rangle\right\}$ [cf. Eq. (10)] through which the system passes under the sequence of measurements. For a quasicontinuous sequence of measurements they form a quasicontinuous trajectory on the Bloch sphere. This trajectory is not closed. It can be argued $[15,20]$ that the natural way of connecting $\left|\psi_{N}\right\rangle$ with $\left|\psi_{N+1}\right\rangle \propto\left|\psi_{0}\right\rangle$ is by drawing the shortest geodesic on the Bloch sphere, which corresponds to a postselected projective measurement at the end of the measurement sequence [cf. the discussion between Eqs. (10) and (12)]. This guarantees that the trajectory is closed. Consider now all trajectories induced when executing the protocol at different $\theta \in[0, \pi]$ for a given $(C, A)$. They form a surface on the Bloch sphere (cf. Fig. 6). We have found numerically 
$|0\rangle$

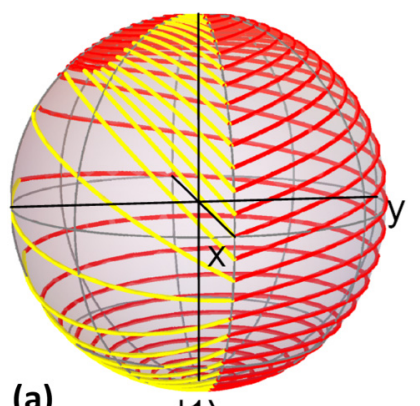

(a) $|0\rangle$

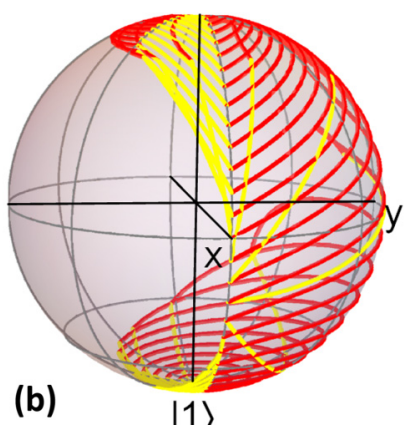

|1)
FIG. 6. Measurement-induced system trajectories $\left\{\left|\psi_{k}\right\rangle\right\}$ on the Bloch sphere for (a) $C=2.3>C_{\text {crit }}$ and (b) $C=1.5<C_{\text {crit }}$, above and below the transition for $A=1$. Different trajectories correspond to the protocols executed at different $\theta$. The red segments correspond to the quasicontinuous sequences $\left\{\left|\psi_{k=0, \ldots, N}\right\rangle\right\}$, while the yellow segments are the shortest geodesics on the Bloch sphere connecting $\left|\psi_{N}\right\rangle$ with $\left|\psi_{N+1}\right\rangle \propto\left|\psi_{0}\right\rangle$. Above the critical measurement strength, the surface wraps around the Bloch sphere, while below, it does not. Confer Fig. 5 of Ref. [20] for the special case of $A=0$.

that for $C>C_{\text {crit }}$ the surface always covers the Bloch sphere, while for $C<C_{\text {crit }}$ it never does. Therefore, the link between the winding number of the measurement-induced phase and the topology of the surface formed by the measurementinduced trajectories exists beyond the case of $A=0$, studied in Ref. [20], notwithstanding the phase not being immediately related to the trajectory (cf. the discussion in Sec. II C). ${ }^{7}$

\section{B. Critical line of the transition}

We have demonstrated in Sec. IV A that there exist special points $\left(C_{\text {crit }}, A_{\text {crit }}, \theta_{\text {crit }}^{(d)}\right)$ where $P_{\left\{r_{k}=0\right\}}^{(d)}\left(\theta_{\text {crit }}^{(d)}\right)=0$. These special

${ }^{7} \mathrm{We}$ emphasize that the two transitions (in the phase winding number and in the topology of the surface formed by the measurement-induced trajectories) always happen concomitantly. In particular, the transition in the surface topology never takes place at $|A|>A_{0}=\pi \sqrt{3} / 2$.

(a)

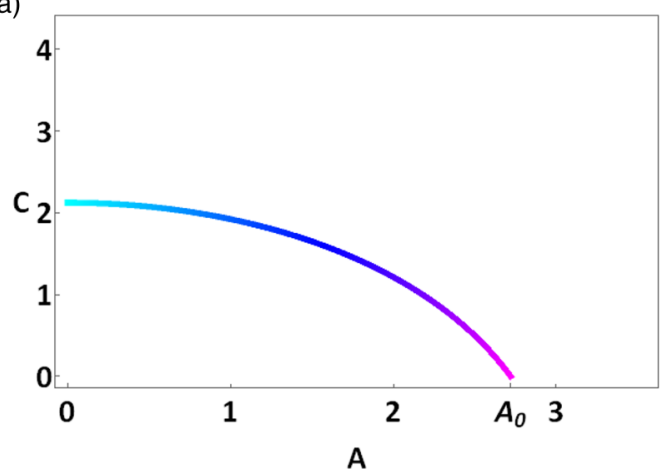

points are associated with phase winding features in the $(C, A)$ plane and with jumps in the winding number $n(C, A)$ [cf. Eq. (59)]. In fact, the set of all these points forms a critical line shown in Fig. 7(a). The derivation of this result is presented in Appendix B.

Note that the critical line for the postselective protocol $\left(\left\{r_{k}=0\right\}\right)$ splits the $(C, A)$ plane into two regions [phases; cf. Fig. 7(a)]. These correspond to two different values of the winding number $n$. The region below the critical line corresponds to $n=0$ (a topologically trivial phase). Indeed, at $(C, A)=(0,0)$ the system is not influenced at all by the measurements leading to $\chi_{\left\{r_{k}=0\right\}}^{(d)}(\theta) \equiv 0$ and $n=0$. Changing the value of a topological index requires passing through a critical point $\left(C_{\text {crit }}, A_{\text {crit }}\right)$ such that $P_{\left\{r_{k}=0\right\}}^{(d)}\left(\theta_{\text {crit }}^{(d)}\right)=0$ at some $\theta=\theta_{\text {crit }}^{(d)}$. Since any point within this region can be accessed from another point by a continuous variation of parameters without crossing the critical line, it follows that $n=0$ throughout this region. Similarly, $(C \rightarrow \infty, A=0)$ corresponds to projective measurement and yields the Pancharatnam phase with $n=$ $-d$. The same connectivity argument implies that this is the value of $n$ throughout the region above the critical line.

We note that the transition only happens for $A \leqslant A_{0}=$ $\pi \sqrt{3} / 2$. While this follows from the solution of the problem (cf. Appendix B), it is instructive to have an intuitive understanding of this fact. For this consider the case of $C=0$. The backaction of an $r_{k}=0$ measurement (and only $r_{k}=0$ are obtained when $C=0$ ) is equivalent to a Hamiltonian evolution for time $\Delta t=1 / N$ in a system with energy gap $\Delta E=$ $-2 A$ [cf. Sec. III B and Eq. (37)]. Then the total evolution under all the measurements in the $N \rightarrow \infty$ limit is equivalent to a Hamiltonian evolution for time $T=N \Delta t=1$ with a continuously evolving Hamiltonian, followed by a projective measurement that ensures the return of the system state to $\left|\psi_{0}\right\rangle$. The rate at which the Hamiltonian parameters are varied is of the order of $v=1 / T=1$. For $A \rightarrow \infty, \Delta E T \sim A v^{-1}=$ $A \gg 1$, so the evolution is adiabatic; the system state follows meticulously the measurement/Hamiltonian axis and acquires the adiabatic Berry phase $\chi_{\left\{r_{k}=0\right\}}^{(d)}(\theta)=\pi d(\cos \theta-1)$ leading to a winding number $n=-d$. For $A<\infty$, the evolution is not adiabatic, implying that the phase will not coincide with the Berry phase (cf. Sec. IIIE 1). At $A=0$, the evolution is

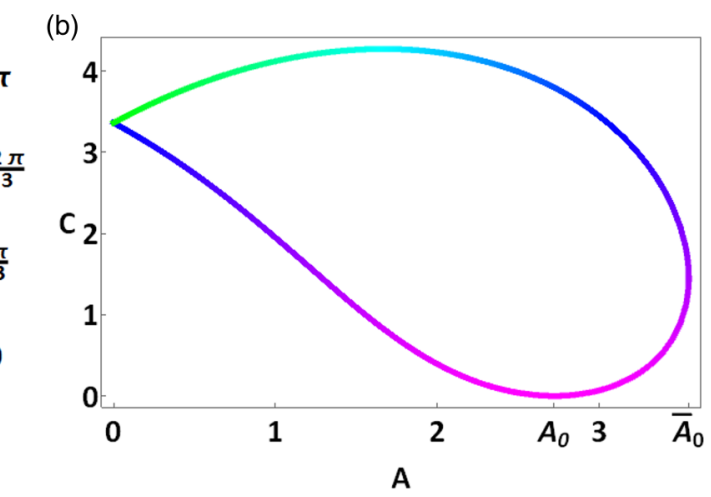

FIG. 7. Critical lines of the topological transitions in the behavior of the weak-measurement-induced phase for the (a) postselective $\left(\chi_{\left\{r_{k}=0\right\}}^{(d=+1)}\right)$ and (b) averaging $\left(\bar{\chi}^{(d=+1)}\right)$ protocols. The lines follow the coordinates $\left(C_{\text {crit }}, A_{\text {crit }}\right)$. The critical polar angle $\theta_{\text {crit }}^{(d=+1)}$ is shown by the color code. For $d=-1$, the transitions take place at the same $\left(C_{\text {crit }}, A_{\text {crit }}\right)$ but at $\theta_{\text {crit }}^{(d=-1)}=\pi-\theta_{\text {crit }}^{(d=+1)}$, as can be inferred from the symmetries discussed in Secs. III C and III D. The behavior at $A<0$ can be inferred too employing those symmetries. 

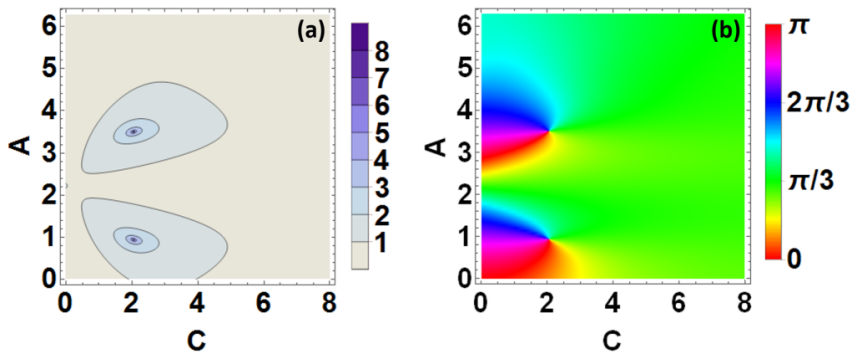

FIG. 8. (a) Dephasing $\alpha^{(+1)}$ and (b) phase $\bar{\chi}^{(+1)}$ [cf. Eq. (21)] at $\theta=3 \pi / 4$ color coded as functions of the measurement strength $C$ and asymmetry $A$ parameters. Note the two singularities at $C \approx 2$, where $\alpha^{(+1)}$ diverges. The phase makes $\pi$ windings around the points of divergent $\alpha^{(+1)}$.

totally nonadiabatic, the system does not have time to sense the change in the Hamiltonian axis, and the acquired phase $\chi_{\left\{r_{k}=0\right\}}^{(d)}(\theta) \equiv 0$, so $n=0$. It is thus clear that there has to be a transition between the two winding numbers at some value of $A=A_{0}$, which is depicted in Fig. 7(a). The required vanishing of the postselection probability $P_{\left\{r_{k}=0\right\}}^{(d)}\left(\theta_{\text {crit }}^{(d)}\right)$ at $A=A_{0}$ is due to the last projective measurement, implying that the state to which the system arrives as a result of the nonadiabatic Hamiltonian evolution is orthogonal to its initial state. ${ }^{8}$

The regimes of the Pancharatnam phase $(C \rightarrow \infty)$ and of the Berry phase $(A \rightarrow \infty)$ share the same topological index $n$. It is thus not surprising that they can be smoothly connected, without crossing any critical lines, as follows from Fig. 7(a).

In the next section we analyze topological transitions of the averaged phase and discuss the qualitative differences from the transitions discussed above.

\section{TOPOLOGICAL TRANSITIONS IN THE AVERAGING PROTOCOL}

The behaviors of the averaged phase $\bar{\chi}^{(d)}$ and dephasing factor $e^{-\alpha^{(d)}}$ bear numerous similarities to the postselected phase $\chi_{\left\{r_{k}=0\right\}}^{(d)}$ and postselection probability $P_{\left\{r_{k}=0\right\}}^{(d)}$. However, there are important qualitative differences that manifest in the topological properties of $\bar{\chi}^{(d)}$.

Similarly to the postselective protocol, the dephasing factor $e^{-\alpha^{(d)}}$ vanishes at specific values $\left(C_{\text {crit }}, A_{\text {crit }}, \theta_{\text {crit }}^{(d)}\right)$ [cf. Fig. 8(a)]. Equivalently, one can say that $\alpha^{(d)}$ diverges at these points. The phase $\bar{\chi}^{(d)}$ makes windings around the points of divergent $\alpha^{(d)}$ [cf. Fig. 8(b)]. However, an important qualitative difference is that $\bar{\chi}^{(d)}$ is defined modulo $\pi$ and not modulo $2 \pi$ as the postselected phases [cf. its definition in Eq. (21)]. This implies that the minimum possible winding is of size $\pi$ [cf. Fig. 8(b)], in contrast to the $2 \pi$ windings of $\chi_{\left\{r_{k}=0\right\}}^{(d)}$ in Fig. 4(b).

The above distinction naturally leads to the fact that the diagram of topological regimes can be richer in the averaging

\footnotetext{
${ }^{8}$ In fact, we find that for any $\left(C_{\text {crit }}, A_{\text {crit }}, \theta_{\text {crit }}^{(d)}\right)$ the final state after weak-measurement-induced evolution $\left|\psi_{N}\right\rangle$ is orthogonal to $\left|\psi_{N+1}\right\rangle \propto\left|\psi_{0}\right\rangle$, while the probability of observing the sequence of $\left\{r_{k=1, \ldots, N}=0\right\}$ is $\left\langle\psi_{N} \mid \psi_{N}\right\rangle \neq 0$.
}

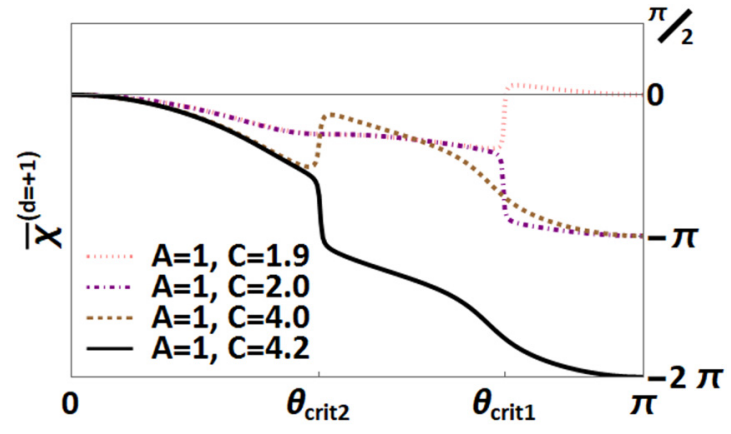

(a)

$\boldsymbol{\theta}$

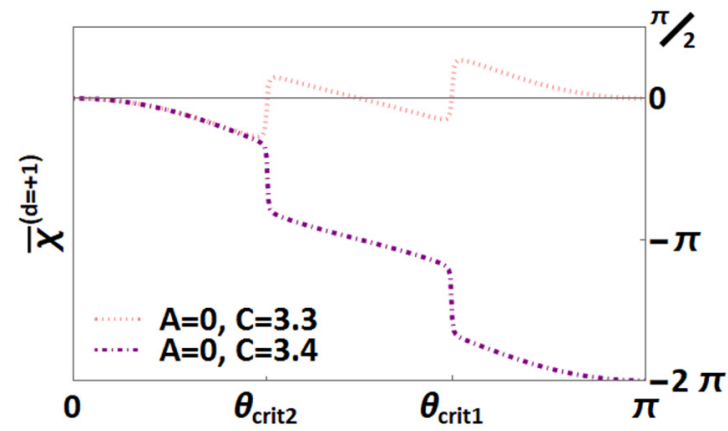

(b)

$\boldsymbol{\theta}$

FIG. 9. Dependence of the averaged phase $\bar{\chi}^{(d=+1)}(\theta)$ on $\theta$ for various $C$ at (a) $A=1$ and (b) $A=0$.

protocol. Similarly to the protocol of the postselected phase, we have $e^{2 i \bar{\chi}^{(d)}-\alpha^{(d)}}=1$ for $\theta=0$ and $\theta=\pi$ [cf. Eqs.(44)(46)]. However, since the averaged phase $\bar{\chi}^{(d)}$ is defined modulo $\pi$ and not $2 \pi$, the good winding number definition is

$$
\bar{n}=\frac{1}{\pi} \int_{0}^{\pi} d \theta \frac{d \bar{\chi}^{(d)}(\theta)}{d \theta}=\frac{\bar{\chi}^{(d)}(\pi)-\bar{\chi}^{(d)}(0)}{\pi} .
$$

Similarly to $n$ in Eq. (59), $\bar{n}$ is also integer valued but demonstrates a larger spectrum of values. Indeed, in the limit of $C \rightarrow \infty \bar{\chi}^{(d)}(\theta)=\pi d(\cos \theta-1)$, implying $\bar{n}=-2 d$; for $C=A=0, \bar{\chi}^{(d)}(\theta) \equiv 0$ and $\bar{n}=0$, yet it is also possible to have $\bar{n}=-d$.

Switching between different values of $\bar{n}(C, A)$ can only happen at $\left(C_{\text {crit }}, A_{\text {crit }}\right)$ for which there exists $\theta_{\text {crit }}^{(d)}$ such that $e^{-\alpha^{(d)}\left(C_{\text {crit }}, A_{\text {crit }}, \theta_{\text {crit }}^{(d)}\right)}=0$ [making the phase $\bar{\chi}^{(d)}\left(C_{\text {crit }}, A_{\text {crit }}, \theta_{\text {crit }}^{(d)}\right)$ undefined]. The set of $\left(C_{\text {crit }}, A_{\text {crit }}\right)$ forms a critical line [Fig. 7(b)] separating the regimes of different $\bar{n}$. Here the critical line splits the $(C, A)$ plane into three regions. The outermost and the innermost regions correspond to $\bar{n}=-2 d$ and $\bar{n}=0$ respectively. The middle one, which was absent in the postselective protocol, corresponds to $\bar{n}=-d$ [cf. Fig. 9(a)]. We emphasize that the $\bar{n}=-d$ region can only be explored with measurements that have non-Hermitian backaction operators (i.e., $A \neq 0$ ).

It is noteworthy that the presence of a middle region is facilitated, yet not dictated, by the definition of the averaged phase modulo $\pi$. Indeed, as a matter of principle, one could define the postselected phase via $P_{\left\{r_{k}\right\}}^{(d)} e^{2 i \chi_{\left\{r_{k}\right\}}^{(d)}}=$ $\left\langle\psi_{0}\left|\mathcal{M}_{N}^{\left(r_{N}\right)} \cdots \mathcal{M}_{1}^{\left(r_{1}\right)}\right| \psi_{0}\right\rangle^{2}$ as opposed to Eq. (12). This would 

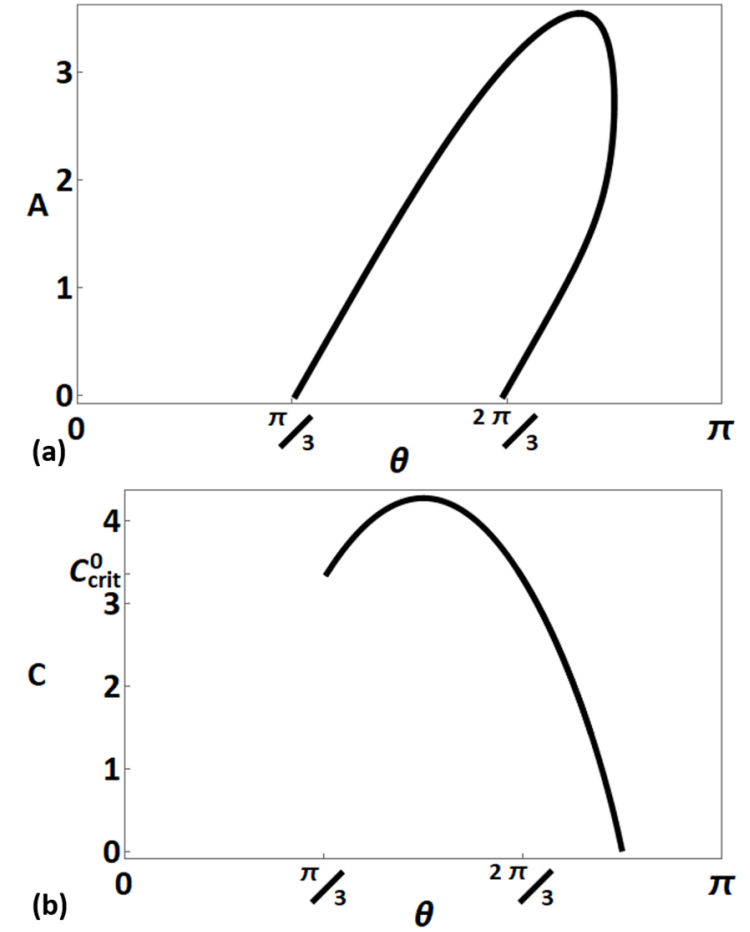

FIG. 10. Projections of the critical line $\left(C_{\text {crit }}, A_{\text {crit }}, \theta_{\text {crit }}^{(d=+1)}\right)$ for the averaged phase [cf. Fig. 7(b)] onto the (a) $(A, \theta)$ and (b) $(C, \theta)$ planes.

imply that $\chi_{\left\{r_{k}\right\}}^{(d)}$ too (not only $\bar{\chi}^{(d)}$ ) is defined modulo $\pi$. Nevertheless, the winding properties of the function $\chi_{\left\{r_{k}\right\}}^{(d)}(\theta)$ would not change with the change of definition. In particular, the winding number $n$ of $\chi_{\left\{r_{k}=0\right\}}^{(d)}(\theta)$ would still acquire only two values, 0 and $-d$ (translating to $\bar{n}=0$ and $-2 d$, respectively). This demonstrates the nontrivial effect of averaging over the readout sequences.

Several other features of the critical line behavior [cf. Fig. 7(b)] are noteworthy. First, note that transitions as a function of $C$ happen only at $A \leqslant \bar{A}_{0} \approx 3.55$. However, the threshold value is different from that in the postselective protocol: $A_{0}<\bar{A}_{0}$.

Second, for any $A \in\left(0, \bar{A}_{0}\right)$ there are two transitions, which correspond to two different critical polar angles $\theta_{\text {crit }}^{(d)}$. At $A=0$ there is only one transition at $C=C_{\text {crit }}^{0} \approx 3.35$ taking $\bar{n}$ from 0 to $-2 d$. The critical polar angles of the two transitions do not merge as $A=0$ is approached. This might be puzzling. The resolution of the puzzle is that at $A=0$, the transition happens as $\bar{\chi}^{(d)}(\theta)$ exhibits two jumps at different values of $\theta$ [cf. Figs. 9(b) and 10(a)].

Third, for $C<C_{\text {crit }}^{0} \approx 3.35$, there are two transitions $(\bar{n}=$ $0 \rightarrow-d$ and $-d \rightarrow-2 d$ ) as a function of $A$ happening at the same value of $\theta$ [cf. Figs. 7, 10(b), and 11(a)]. For $C>$ $C_{\text {crit }}^{0}$, there are again two distinct $A_{\text {crit }}$, yet now the transitions correspond to $\bar{n}=-2 d \rightarrow-d$ and $-d \rightarrow-2 d$ and happen at two different values of $\theta$ [cf. Fig. 11(b)].

Finally, both the averaged and the postselected transition have the same $\left(A_{\text {crit }}, \theta_{\text {crit }}^{(d=+1)}\right)$ at $C=0$. This is easy to understand, as at $C=0$ essentially no measurement takes place and there is no difference between $\bar{\chi}^{(d)}(\theta)$ and $\chi_{\left\{r_{k}\right\}}^{(d)}(\theta)$ : The only readout sequence that can be obtained is $\left\{r_{k}=0\right\}$ (cf.

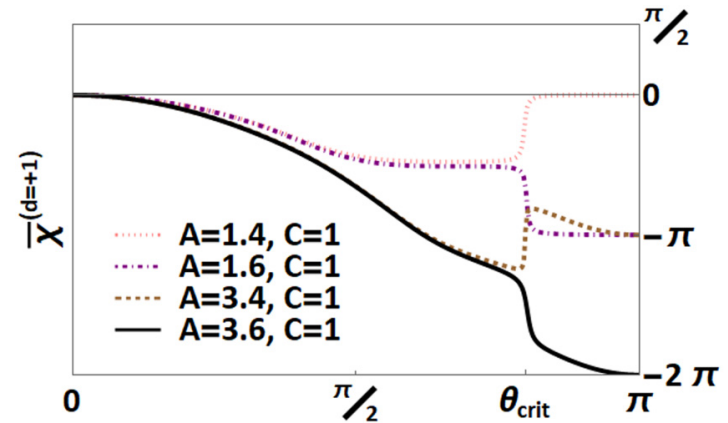

(a)

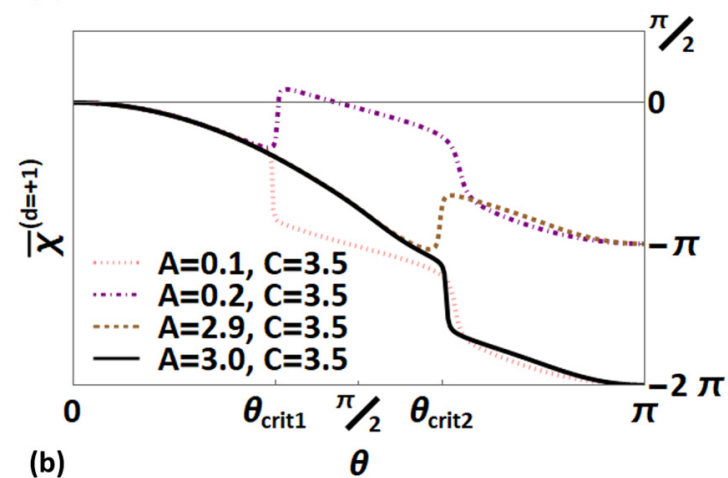

FIG. 11. Dependence of the averaged phase $\bar{\chi}^{(d=+1)}(\theta)$ on $\theta$ for various $A$ at (a) $C=1$ and (b) $C=3.5$.

Sec. III E 3). However, already at arbitrarily small $C$, the critical lines of the two protocols behave in drastically different ways.

In Sec. IV A we linked the topological transitions in the postselective protocol to a change in the collective properties of measurement-induced state trajectories (cf. Fig. 6). Establishing such a connection for the averaging protocol is not straightforward since averaging over the detector readouts in the phase definition [cf. Eq. (21)] implies that many measurement-induced trajectories are involved for each value of protocol parameters. Recently, it has been predicted theoretically [36] and observed experimentally [37] that measurement-induced dynamics can exhibit one or multiple optimal (most probable) quantum trajectories depending on the system parameters. Further, in the case of multiple optimal trajectories the system may exhibit chaotic behavior [38]. It would be interesting to investigate whether transitions between these different regimes exist in our system and, if they do, whether they are linked to the topological transitions we report here.

\section{EXPERIMENTAL IMPLEMENTATION}

In this section we discuss conceptual experimental setups that enable observing the measurement-induced phases defined and investigated above. We pay particular attention to some practical aspects of measuring the averaged phase.

\section{A. Interferometric detection schemes}

In order to measure the effects discussed in the previous sections, it is crucial to have the ability to access the 

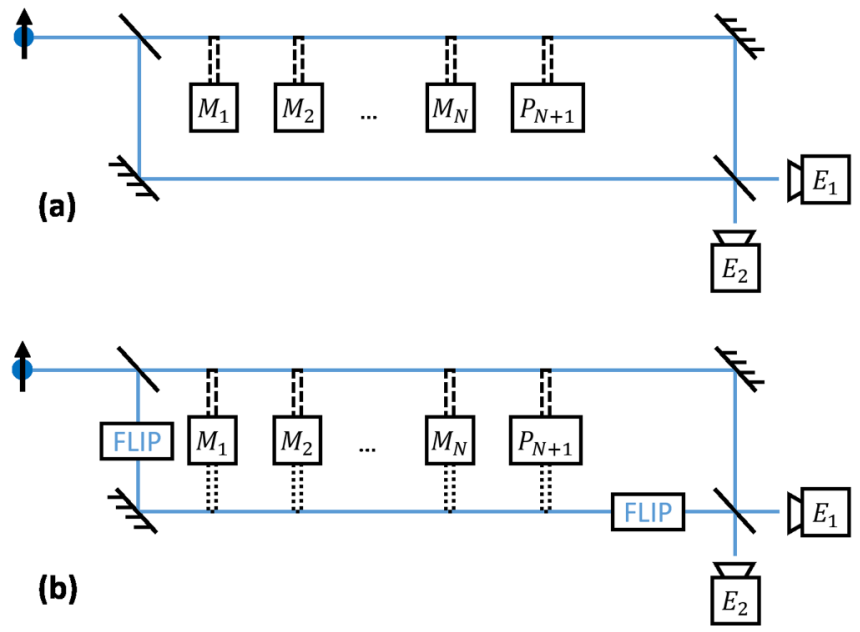

FIG. 12. Interferometry setups for observing measurementinduced phases (a) $\chi_{\left\{r_{k}=0\right\}}$ and (b) $\bar{\chi}$ [cf. Eqs. (12) and (13)]. A particle, whose spin represents the measured system, flies through the interferometer. Weak measurements of its spin are denoted by $M_{k=1, \ldots, N}$, while the last postselected projective measurement is denoted by $P_{N+1}$. The protocol of (b) for detecting the averaged phase involves two special features. First, the particle spin is flipped in one arm as indicated by the "FLIP" boxes. Second, the detectors interact with the two arms via different Hamiltonians (see Appendix $\mathrm{C}$ for details).

measurement-induced phases. Here we define two conceptual setups that facilitate measurement of the postselected $\chi_{\left\{r_{k}=0\right\}}$ and the averaged $\bar{\chi}$ phases [defined in Eqs. (12) and (13), respectively]. The setup for measuring $\chi_{\left\{r_{k}=0\right\}}$ is shown in Fig. 12(a). A particle with spin in state $\left|\psi_{0}\right\rangle$ enters a MachZehnder interferometer and is split into two arms. In one arm, the particle is subjected to a sequence of weak measurements and one projective measurement (implementing the protocol described in Sec. III). In the other arm, the particle flies through unaffected. As a result, the state of the particle and detectors just before the particle reaches the final beam splitter is

$$
\begin{aligned}
|\Psi\rangle= & \frac{1}{\sqrt{2}}\left|\psi_{0}\right\rangle|-1\rangle_{a} \prod_{k=1}^{N+1}\left|r_{k}=0\right\rangle_{D_{k}}+\frac{1}{\sqrt{2}} \sum_{\left\{r_{k}\right\}} \delta_{r_{N+1}, 0} \\
& \times\left|\psi_{0}\right\rangle|+1\rangle_{a}\left\langle\psi_{0}\left|\mathcal{M}_{N}^{\left(r_{N}\right)} \cdots \mathcal{M}_{1}^{\left(r_{1}\right)}\right| \psi_{0}\right\rangle \prod_{k=1}^{N+1}\left|r_{k}\right\rangle_{D_{k}},
\end{aligned}
$$

where $| \pm 1\rangle_{a}$ denotes the particle being in the upper/lower arm and $\left|r_{k}\right\rangle_{D_{k}}$ is the state of the $k$ th detector. We have accounted here for the fact that as the particle is flying through the lower arm, the detectors remain in their initial states $\left|r_{k}=0\right\rangle$ (which are the initial states of the detectors in the measurement model described in Sec. III A). As a result, the intensities observed at the interferometer exits $E_{1,2}$ will be

$$
\begin{aligned}
I_{1,2}= & \frac{I_{0}}{2}\left(\frac{1}{2}+\frac{1}{2} \sum_{\left\{r_{k}\right\}}\left|\left\langle\psi_{0}\left|\mathcal{M}_{N}^{\left(r_{N}\right)} \cdots \mathcal{M}_{1}^{\left(r_{1}\right)}\right| \psi_{0}\right\rangle\right|^{2}\right. \\
& \left. \pm \operatorname{Re}\left\langle\psi_{0}\left|\mathcal{M}_{N}^{(0)} \cdots \mathcal{M}_{1}^{(0)}\right| \psi_{0}\right\rangle\right)
\end{aligned}
$$

where $I_{0}$ is the intensity of the incoming particle beam; the second term on the right-hand side of Eq. (62) is less than $\frac{1}{2}$ as it accounts for the loss of particles due to discarding the runs in which the last projective measurement yields $r_{N+1}=1$; the last (interference) term gives $\sqrt{P_{\left\{r_{k}=0\right\}}} e^{i \chi_{\left.\mid r_{k}=0\right\}} \text {. This scheme }}$ thus enables the observation of the measurement-induced phase for the readout sequence $\left\{r_{k}=0\right\}$. The scheme relies crucially on the fact that the readouts $r_{k}=0$ correspond to the detector initial state being unchanged.

The setup of Fig. 12(b) shows how the averaged phase $\bar{\chi}$ can be measured. Now the particle interacts with the detectors in both arms. Moreover, the $k$ th measurement is performed in both arms by the same physical detector that is later read out, thus ensuring that the readout $r_{k}$ is the same in both arms. However, measuring $e^{2 i \bar{\chi}}$ as defined in Eq. (13) through interference requires that for each readout sequence $\left\{r_{k}\right\}$ the

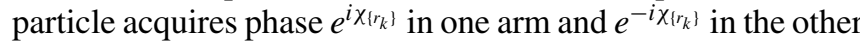
arm. In order to achieve that, we propose to flip the particle spin when it enters and exits the lower arm and in addition to use somewhat different particle-detector interaction Hamiltonians in the two arms. We give the details of the procedure in Appendix C. The resulting intensities at the interferometer exits $E_{1,2}$ are

$$
\begin{aligned}
I_{1,2}= & \frac{I_{0}}{2}\left(\sum_{\left\{r_{k}\right\}}\left|\left\langle\psi_{0}\left|\mathcal{M}_{N}^{\left(r_{N}\right)} \cdots \mathcal{M}_{1}^{\left(r_{1}\right)}\right| \psi_{0}\right\rangle\right|^{2}\right. \\
& \left. \pm \operatorname{Re} \sum_{\left\{r_{k}\right\}}\left(\left\langle\psi_{0}\left|\mathcal{M}_{N}^{\left(r_{N}\right)} \cdots \mathcal{M}_{1}^{\left(r_{1}\right)}\right| \psi_{0}\right\rangle\right)^{2}\right),
\end{aligned}
$$

where the first term accounts for the particle loss in the last projective measurement postselection and the interference term is exactly $e^{2 i \bar{\chi}-\alpha}$ in Eq. (13). ${ }^{9}$

We stress that while for the reasons of theoretical simplification we have considered the limit of the number of measurements $N \rightarrow \infty$ in the above sections, essentially the same physics of asymmetric behavior of the phases, the postselection probability, and the dephasing parameter will appear for sequences of measurements with any $N \geqslant 2$. Furthermore, the points of vanishing postselection probability and the singularities of the dephasing parameter will be related to the topological transitions in the phase behavior for finite $N$ too. However, some specific features (such as the shape of the critical lines) will be modified in the case of finite $N$. In particular, the results will depend periodically on $A$ with the period being $\pi N$.

\section{B. Practicalities of averaging over readout sequences in experiment}

The definition of the averaged phase $\bar{\chi}$ in Eq. (13) requires averaging the postselected phases $\chi_{\left\{r_{k}\right\}}$ over all possible readout sequences $\left\{r_{k}\right\}$ weighted with appropriate probabilities. However, this does not correspond to the interferometric procedure for measuring $\bar{\chi}$ outlined above. Indeed, a particle

\footnotetext{
${ }^{9}$ Equation (63) is valid for arbitrary $N$ for the protocol defined in Sec. III. However, Eq. (63) does not apply to protocols with other choices of the measurement axes $\mathbf{n}_{k}^{(s)}$ and/or the initial state $\left|\psi_{0}\right\rangle$.
} 


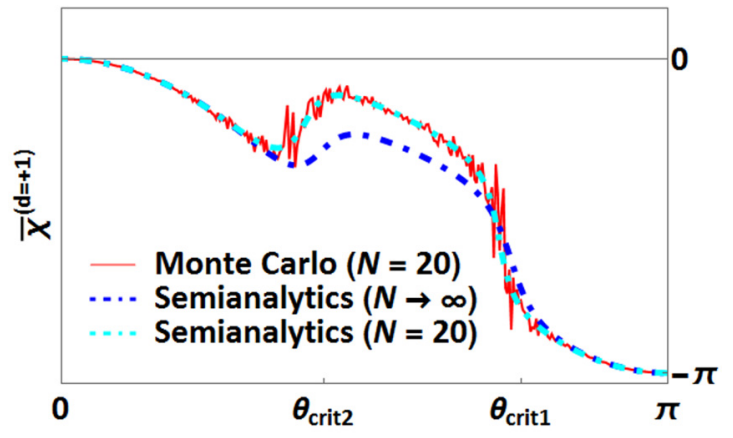

(a)

$\theta$

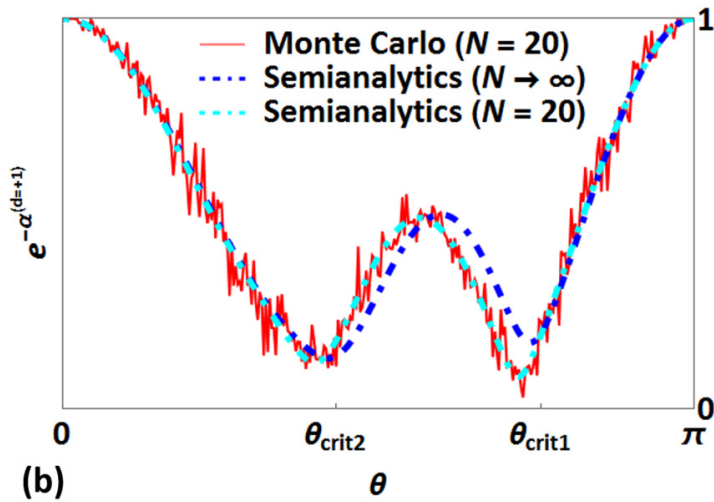

FIG. 13. Comparison of Monte Carlo simulations for (a) the averaged phase $\bar{\chi}^{(d=+1)}$ and (b) the dephasing parameter $\alpha^{(d=+1)}$ with the results obtained using the semianalytical method of Sec. IIID. The number of Monte Carlo samples (readout sequence realizations $\left\{r_{k}^{(i)}\right\}$ ) used is $N_{\mathrm{rs}}=100$. The number of measurements in the sequence is denoted by $N$. The plots correspond to $C=3$ and $A=1$.

flying through the interferometer will yield a specific readout sequence $\left\{r_{k}\right\}$ and a specific phase $\chi_{\left\{r_{k}\right\}}$ with probability $P_{\left\{r_{k}\right\}}$. The next particle will again yield a random readout sequence $\left\{r_{k}^{\prime}\right\}$ and so on. Therefore, the actual measurement procedure is identical to a Monte Carlo sampling of the readout sequences rather than systematic summing over them. The number of such sequences scales as $2^{N}$ with the number of measurements $N$. Sampling such a large number of sequences (for large $N$ ) is impossible. However, the probability of a specific sequence $\left\{r_{k}\right\}$ determines both the frequency of obtaining this sequence and its contribution to the sum, rendering it possible to obtain an accurate estimate of $\bar{\chi}$ with a moderate number of experimental runs.

We have performed a Monte Carlo study that simulates the sampling of $\left\{r_{k}\right\}$ in the experiment. Namely, we randomly generated the readout sequences according to the algorithm outlined in Ref. [20] and calculated

$$
\left\langle e^{\left.2 i \chi_{\left\langle r_{k}\right\}}\right\rangle}=\frac{1}{N_{\mathrm{rs}}} \sum_{i=1}^{N_{\mathrm{rs}}} e^{2 i \chi_{\left\langle r_{k}^{(i)}\right.}},\right.
$$

where $N_{\text {rs }}=100$ readout sequences $\left\{r_{k}^{(i)}\right\}$ were generated for sequences of $N=20$ measurements. A comparison of the Monte Carlo simulations to the results obtained using the method of Sec. III D is shown in Fig. 13. The Monte Carlo curves reproduce the behavior for $N \rightarrow \infty$ qualitatively and closely follow the exact result for $N=20$. We therefore conclude that the experimental procedure does allow one to probe the physics discussed above with reasonable accuracy. Although pinpointing the exact locations of the critical lines of the topological transitions [where the terms in the sum in Eq. (13) accurately cancel out to yield $e^{2 i \bar{\chi}-\alpha}=0$ ] may require a large number of experimental runs, establishing the existence of several topological sectors with different winding numbers $\bar{n}$ can be done without accumulating too large a statistics.

\section{CONCLUSION}

We have performed a detailed investigation of measurement-induced phase factors. Our theory puts forth two classifications of such phases: dynamical vs geometrical phases and components which are symmetric/antisymmetric with respect to the reversal of the measurement sequence. Importantly, we have shown, based on general considerations and on a specific example, that these two classifications do not coincide.

We have demonstrated our theoretical framework via analyzing a specific protocol, calculating postselected and averaged measurement-induced phases, and investigating their dependence on various measurement parameters. We have shown that the projective-measurement-induced Pancharatnam phase and the Berry phase induced by adiabatic Hamiltonian evolution can be viewed as two (out of several) limiting cases of the phases induced by quasicontinuous sequences of weak measurements.

We have found and investigated topological transitions pertaining to measurement-induced phases. We have found the phase diagram of different topological regimes and discussed its distinctive features. While we have investigated topological transitions for a specific protocol, the generality of our considerations may lead one to believe that such transitions are a generic feature of measurement-induced phases, avoiding the need to refer to a specific measurement model or phase-inducing protocol. Nevertheless, the details of the phase diagram may depend on the specific protocol and measurement class.

Finally, we have proposed experimental setups facilitating the observation of weak-measurement-induced phases and the study of the effects discussed in this work. We believe that weak-measurement-induced phase factors present a rich playground that may be important for understanding topological phases of matter in open quantum systems.

\section{ACKNOWLEDGMENTS}

We thank V. Gebhart for useful discussions. We acknowledge funding from the Deutsche Forschungsgemeinschaft (German Research Foundation) through Project No. 277101999, TRR 183 (Project C01), and Projects No. EG 96/13-1, GO 1405/6-1, and MI 658/10-2, as well as from the Israel Science Foundation.

\section{APPENDIX A: INVESTIGATION OF DIFFERENT SCALING REGIMES}

As mentioned in Sec. III B, taking the limit $N \rightarrow \infty$ in our protocols requires adjusting (scaling) the measurement 
parameters $g$ and $\theta^{(D)}$ (cf. Sec. III A), performing this as a function of $N$. Here we explore the possible ways of scaling. We show that the only nontrivial scaling regime corresponds to the one presented in Sec. III B.

One can understand the need for scaling of the measurement parameters in the quasicontinuous limit $(N \rightarrow \infty)$ from the following consideration. Arrange the measurements into $j$ sets $(N \gg j \gg 1)$, that is, measurements $k=1, \ldots, N / j$ form one set, $k=N / j+1, \ldots, 2 N / j$ are the second set, etc. Within each set, the axes of the measured system observables are clustered at $\varphi_{k} \approx \tilde{\varphi}_{k}=2 \pi d\lfloor(k-1) j / N\rfloor / j$, where $\lfloor(k-1) j / N\rfloor$ is the set number; here $\lfloor x\rfloor$ is the floor function. The spread of the actual $\varphi_{k}$ from $\tilde{\varphi}_{k}$ is $O(2 \pi d / j)$ and can be made arbitrarily small in the limit $N \rightarrow \infty$ via taking arbitrarily large values of $j$. Then this set of weak measurements can be interpreted as a single projective measurement with the appropriate axis $\tilde{\mathbf{n}}_{k}^{(s)} \approx \mathbf{n}_{k}^{(s)}$. Indeed, if all measurements in a set yield $r_{k}=0$, the backaction on the system state can be described by $\prod_{k} R^{-1}\left(\mathbf{n}_{k}^{(s)}\right) M^{(0)} R\left(\mathbf{n}_{k}^{(s)}\right) \approx$ $R^{-1}\left(\tilde{\mathbf{n}}_{k}^{(s)}\right)\left(M^{(0)}\right)^{N / j} R\left(\tilde{\mathbf{n}}_{k}^{(s)}\right)$, with $M^{(0)}$ and $R\left(\mathbf{n}^{(s)}\right)$ defined in Eqs. (29) and (31), respectively. Therefore, $\left(M^{(0)}\right)^{N / j}$ plays the role of the effective backaction matrix $\tilde{M}^{(0)}$. In the limit $N / j \rightarrow \infty$, with fixed measurement parameters $g, \theta^{(D)}$, and $\varphi^{(D)}=-\pi / 2$,

$$
\tilde{M}^{(0)}=\left(M^{(0)}\right)^{N / j}=\left(\begin{array}{ll}
1 & 0 \\
0 & 0
\end{array}\right)
$$

unless $\sin g \sin \theta^{(D)}=0$. Therefore, for generic measurement parameters, a set of measurements all yielding $r=0$ is equivalent to a single projective measurement yielding $r=0$. If at least one measurement in the set yields $r=1$, the form of $M^{(1)}$ ensures that the system state is projected onto the $\downarrow$ eigenstate of $\tilde{\mathbf{n}}_{k}^{(s)} \cdot \boldsymbol{\sigma}^{(s)}$, again making the backaction identical to that of a single projective measurement [up to errors $O(2 \pi d / j)$ ]. Since the backaction also determines the probabilities of the outcomes (cf. Sec. II A), one concludes that the original protocol is equivalent to a quasicontinuous sequence of strong measurements. The latter yields the Pancharatnam phase.

A nontrivial scaling limit thus requires $\lim _{N / j \rightarrow \infty} \mid \cos g+$ $\left.i \sin g \cos \theta^{(D)}\right|^{N / j}>0$. Since the scaling of the measurement parameters should not depend on the number of groups $j$ but only on $N$, this is equivalent to $\lim _{N \rightarrow \infty} \mid \cos g+$ $\left.i \sin g \cos \theta^{(D)}\right|^{N}>0$. Similarly, we require $\lim _{N \rightarrow \infty} \mid \cos g+$ $\left.i \sin g \cos \theta^{(D)}\right|^{N}<1$ as the opposite would imply that there is zero probability of obtaining an $r=1$ readout, making the evolution completely deterministic (equivalent to Hamiltonian evolution). We allow the scaling of $g=C^{\prime} N^{-a}$ and $\theta^{(D)}=\pi / 2-A^{\prime} N^{-b}$ with $a, b \geqslant 0$. The above requirements then imply $a=\frac{1}{2}$. With this choice, the single measurement $r=0$ backaction matrix becomes

$$
\begin{aligned}
M^{(0)} & =\left(\begin{array}{cc}
1 & 0 \\
0 & 1-\frac{C^{\prime 2}}{2 N}+i \frac{C^{\prime} A^{\prime}}{N^{1 / 2+b}}+O\left(N^{-3 b}, N^{-b-3 / 2}\right)
\end{array}\right) \\
& =\left(\begin{array}{cc}
1 & 0 \\
0 & \exp \left(-2 \frac{C+i A N^{1 / 2-b}}{N}+O\left(N^{-3 b}, N^{-b-3 / 2}\right)\right)
\end{array}\right),
\end{aligned}
$$

where in the last step we defined $C=C^{2} / 4$ and $A=$ $-A^{\prime} C^{\prime} / 2$.
We now need to choose the appropriate scaling of $b$. Again, a qualitative consideration is useful here. Note that

$$
\begin{aligned}
M^{(0)} & =\left(\begin{array}{cc}
1 & 0 \\
0 & \exp \left(-2 \frac{C+i A N^{1 / 2-b}}{N}\right)
\end{array}\right) \\
& =\left(\begin{array}{cc}
1 & 0 \\
0 & \exp \left(-2 \frac{C}{N}\right)
\end{array}\right) e^{-i H \Delta t},
\end{aligned}
$$

where $H=B\left(\mathbb{I}-\sigma_{z}^{(s)}\right)$ with $B=A N^{1 / 2-b}$ and $\Delta t=1 / N$. In other words, a measurement of the class we consider, when yielding $r=0$, can be decomposed into a Hamiltonian evolution over time $\Delta t$, followed by a measurement with a Hermitian backaction matrix. Let us first ignore the measurement (set $C=0$ ). Then the readout $r=0$ is implied since $M^{(1)}=0$ [cf. Eq. (30)]. The evolution is a quasicontinuous Hamiltonian evolution, with the magnetic field axis changing its direction by $\Delta \varphi=2 \pi d \Delta t$ after every time interval $\Delta t$. In the limit $\Delta t \rightarrow 0$ (or equivalently $N \rightarrow \infty$ ), this becomes a continuously evolving Hamiltonian with its axis changing at the rate $d \varphi / d t=2 \pi d$ and the energy gap $2 A N^{1 / 2-b}$. Therefore, for $b<\frac{1}{2}$, the adiabatic theorem applies to the system as the gap size is infinitely large; the system state will then meticulously follow the measurement axis. Now, introducing $C>0$ does not modify the picture much as the corresponding part of the backaction pulls the state closer towards the measurement axis, to which the state is close anyway. Numerical investigation shows that this qualitative consideration is correct (cf. Fig. 14). Moreover, one can analytically show that for $b<\frac{1}{2}$, the probability of getting all measurement outcomes $r_{k}=0$, $P_{\left\{r_{k}=0\right\}}^{(d)}=1$, while the phase $\chi_{\left\{r_{k}=0\right\}}^{(d)}=-\pi d(1-\cos \theta)$ coincides with the Berry phase for the corresponding Hamiltonian evolution. The regime of $b>\frac{1}{2}$ is also not interesting for us as the non-Hermitian part becomes insignificant when $N \rightarrow \infty$ and the problem reduces to that investigated in Ref. [20].

We conclude that the nontrivial scaling regime corresponds to $a=b=\frac{1}{2}$ with $g=\sqrt{4 C / N}$ and $\theta^{(D)}=\pi / 2+A / \sqrt{C N}$. This is the regime presented in Sec. III B.

\section{APPENDIX B: FINDING THE CRITICAL LINE FOR THE POSTSELECTIVE PROTOCOL}

Here we look for the parameters $\left(C_{\text {crit }}, A_{\text {crit }}, \theta_{\text {crit }}^{(d)}\right)$ where $P_{\left\{r_{k}=0\right\}}^{(d)}=0$. We focus on $A \geqslant 0$. The critical line at $A<0$ can be inferred using the symmetries discussed at the end of Sec. III C. Equation (40) implies that $P_{\left\{r_{k}=0\right\}}^{(d)}=0$ is equivalent to

$$
\cosh \tau+Z \frac{\sinh \tau}{\tau}=0 .
$$

The real and imaginary parts of this equation represent two equations for three parameters $C, A$, and $\theta$. Therefore, one expects the solution to represent a line in the space of these three parameters.

We first observe numerically that if Eq. (B1) holds at some $\left(C_{\text {crit }}, A_{\text {crit }}, \theta_{\text {crit }}^{(d)}\right)$, then the imaginary part of the lefthand side of Eq. (B1) vanishes at $\left(C, A_{\text {crit }}, \theta_{\text {crit }}^{(d)}\right)$ for any $C$ [cf. Fig. 15(a)]. Assuming that this observation is exact, we extract the relation between $A_{\text {crit }}$ and $\theta_{\text {crit }}^{(d)}$ as follows. We expand Eq. (B1) at $C \rightarrow \infty$ and demand that its imaginary 

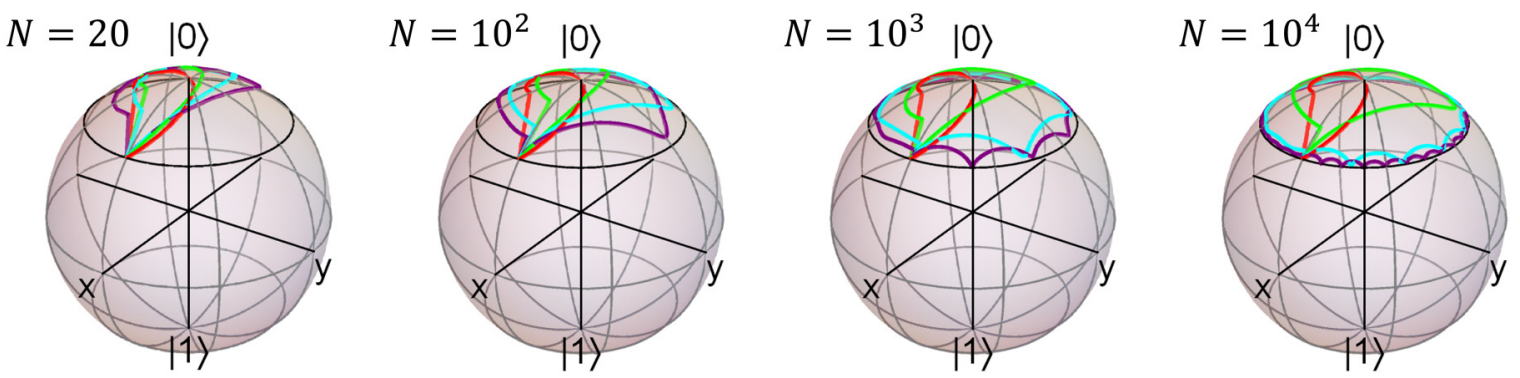

FIG. 14. Trajectories of the quantum state $\left|\psi_{k}\right\rangle$ [Eq. (10)] on the Bloch sphere in various scaling limits. Plotted are the trajectories induced by sequences of $N$ measurements around the parallel corresponding to $\theta=\pi / 4$ (black) with all measurements yielding readouts $r_{k}=0$. The backaction matrix $M^{(0)}$ is given in Eq. (A3). The trajectories are plotted for $b=0$ (purple), 0.1 (cyan), 0.3 (green), and 0.5 (red) using $C=1, A=1$, and $d=+1$. All the trajectories with $b<\frac{1}{2}$ converge towards the parallel line (i.e., the trajectory of the measurement axes) as $N \rightarrow \infty$. This does not happen for $b=\frac{1}{2}$. Moreover, the trajectory seems not to change with increasing $N$, suggesting that already at $N=20$ measurements the measurement-induced trajectory has converged.

part vanishes to the leading order in $C$, obtaining the condition

$$
A_{\text {crit }}+\pi d \cos \theta_{\text {crit }}^{(d)}=0 .
$$

It is now straightforward to verify that this condition indeed implies that the imaginary part of the left-hand side of Eq. (B1) vanishes. Indeed, when Eq. (B2) holds, $Z=C$ and $\tau=\sqrt{Z^{2}-\pi^{2} \sin ^{2} \theta_{\text {crit }}^{(d)}}$ is either purely real or purely imaginary; therefore, $Z, \cosh \tau$, and $\sinh \tau / \tau$ are all real.

Switching to the real part of Eq. (B1), we rewrite the equation as

$$
\tau \operatorname{coth} \tau=-Z \text {. }
$$

Squaring the equation and recalling that

$$
Z=C_{\text {crit }}=\sqrt{\tau^{2}+\pi^{2} \sin ^{2} \theta_{\text {crit }}^{(d)}}
$$

(where the sign of the square root is dictated by the fact that $C \geqslant 0$ ), we obtain

$$
\frac{\tau^{2}}{\sinh ^{2} \tau}=\pi^{2} \sin ^{2} \theta_{\text {crit }}^{(d)} \Longleftrightarrow \frac{\tau}{\sinh \tau}= \pm \pi \sin \theta_{\text {crit }}^{(d)} .
$$

Recall that only $\tau$ being real or imaginary are the cases of interest for us. For real $\tau, \tau / \sinh \tau \geqslant 0$, dictating the choice of + in Eq. (B5). Using this and Eq. (B4), we rewrite Eq. (B1) as

$$
\begin{aligned}
\cosh \tau+\frac{\sqrt{\tau^{2}+\pi^{2} \sin ^{2} \theta_{\text {crit }}^{(d)}}}{\pi \sin \theta_{\text {crit }}^{(d)}} & =\cosh \tau+\sqrt{\sinh ^{2} \tau+1} \\
& =2 \cosh \tau \geqslant 2>0,
\end{aligned}
$$

implying that there are no solutions with real $\tau$.

We thus look for solutions with $\tau=i b, b \in \mathbb{R}$. Since $\tau$ and $-\tau$ are equivalent in Eq. (B1), we choose $b \geqslant 0$ without loss of generality. Then Eq. (B5) becomes

$$
\frac{b}{\sin b}= \pm \pi \sin \theta_{\text {crit }}^{(d)} \Longleftrightarrow \frac{\sin b}{b}= \pm \frac{1}{\pi \sin \theta_{\text {crit }}^{(d)}} .
$$

The right-hand side of Eq. (B7) is either $\geqslant 1 / \pi$ or $\leqslant-1 / \pi$. One sees from Fig. 15 (b) that $|\sin b / b| \geqslant 1 / \pi$ only for $b \leqslant$ $b_{c}<\pi$. In this range of $b, \sin b / b>0$; therefore, one has to choose + in Eq. (B7). No solution of Eq. (B7) with + is a

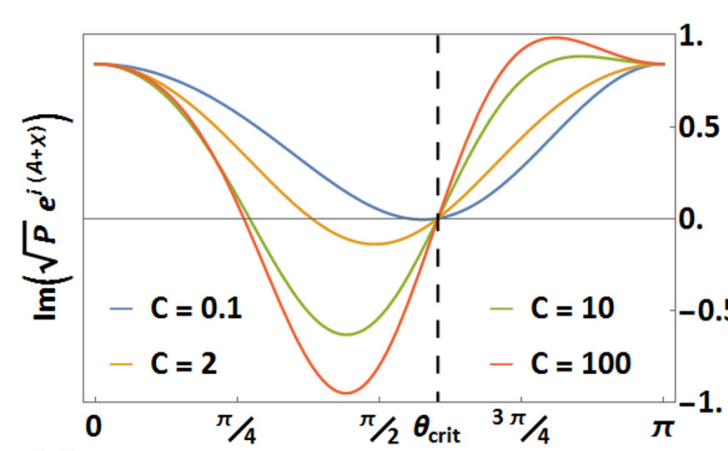

(a) $\boldsymbol{\theta}$

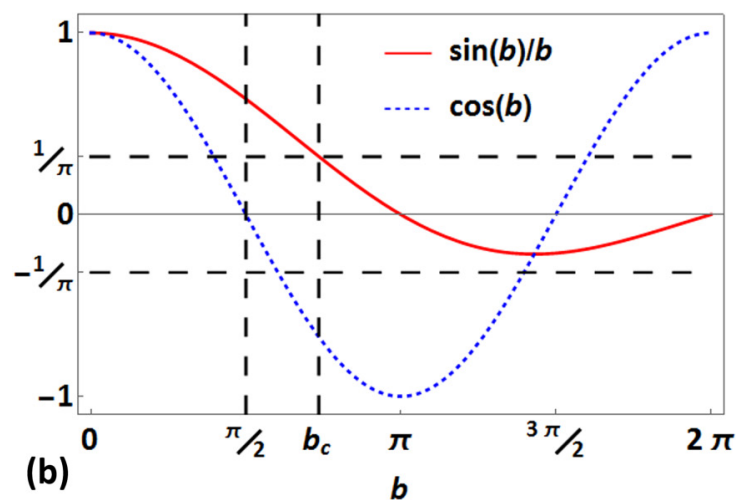

FIG. 15. (a) Imaginary part $\operatorname{Im}\left(\sqrt{P_{\left\{r_{k}=0\right\}}^{(d=+1)}} e^{i \chi_{\left\{r_{k}=0\right\}}^{(d=+1)}+i A}\right) \equiv \operatorname{Im}\left(\sqrt{P} e^{i(A+\chi)}\right)$ as a function of $\theta$ for various $C$ at $A=1$. One can check that the probability $P_{\left\{r_{k}=0\right\}}^{(=+1)}=0$ at $\left(C_{\text {crit }} \approx 1.925, A_{\text {crit }}=1, \theta_{\text {crit }} \approx 1.894\right)$. The imaginary part vanishes at $\theta=\theta_{\text {crit }}$ for any $C$. (b) Dependence of sin $b / b$ and $\cos b$ on $b$. Here $b_{c}$ is such that $\sin b_{c} / b_{c}=1 / \pi$. 
solution of Eq. (B1). Indeed,

$$
\begin{aligned}
\cosh \tau+Z \frac{\sinh \tau}{\tau} & =\cos b+\frac{\sin b}{b} \sqrt{\pi^{2} \sin ^{2} \theta_{\text {crit }}^{(d)}-b^{2}} \\
& =\cos b+\sqrt{1-\frac{b^{2}}{\pi^{2} \sin ^{2} \theta_{\text {crit }}^{(d)}}} \\
& =\cos b+\sqrt{1-\sin ^{2} b} \\
& =\cos b+|\cos b| .
\end{aligned}
$$

Therefore, only the $b$ yielding $\cos b<0$ will be actual solutions of Eq. (B1). One can see from Fig. 15(b) that these are $b \in\left[\pi / 2 ; b_{c}\right]$.

At $b=b_{c}, \sin b / b=1 / \pi$, implying $\theta_{\text {crit }}^{(d)}=\pi / 2$, while $b=$ $\pi / 2$ implies $\sin \theta_{\text {crit }}^{(d)}=\frac{1}{2}$. Taking into account Eq. (B2), one sees that for $A \geqslant 0$ only $\theta_{\text {crit }}^{(d=+1)} \in[\pi / 2 ; 5 \pi / 6]$ are allowed [which implies, again through Eq. (B2), that only $A_{\text {crit }} \leqslant A_{0}=$ $\pi \sqrt{3} / 2$ are possible]. Now one can construct the critical line. For each $\theta_{\text {crit }}^{(d=+1)}$ in this range, $A_{\text {crit }}$ is found from Eq. (B2); at the same time one solves Eq. (B7) with + numerically to find $b$, which then yields $C_{\text {crit }}=\sqrt{\pi^{2} \sin ^{2} \theta_{\text {crit }}^{(d)}-b^{2}}$. The resulting critical line is shown in Fig. 7.

We note that the arguments presented above rely on the initial assumption that $P_{\left\{r_{k}=0\right\}}^{(d)}$ can only turn to 0 when Eq. (B2) holds. Abandoning this assumption, there might be in principle additional critical sets $\left(C_{\text {crit }}, A_{\text {crit }}, \theta_{\text {crit }}^{(d)}\right)$ that are not included in these considerations. Our numerical investigation, though, showed no evidence of such points.

\section{APPENDIX C: DETAILS ON THE AVERAGED PHASE DETECTION SCHEME}

The averaged phase detection setup shown in Fig. 12(b) involves detectors interacting with two arms of the interferometer via different Hamiltonians. Denoting the upper/lower arm as $a= \pm 1$, we write

$$
\begin{aligned}
H_{\mathrm{int}}^{(a)}= & -\frac{\lambda(t)}{2}\left[1-a\left(\mathbf{n}^{(s)} \cdot \boldsymbol{\sigma}^{(s)}\right)\right] \\
& \times\left(n_{y}^{(D)} \sigma_{y}^{(D)}+a n_{x}^{(D)} \sigma_{x}^{(D)}+a n_{z}^{(D)} \sigma_{z}^{(D)}\right) .
\end{aligned}
$$

For the upper arm, $a=+1$, which reduces to Eq. (25). For the lower arm, $a=-1$, which leads to two modifications: The signs of $n_{x, z}^{(D)}$ are changed and $-\mathbf{n}^{(s)} \cdot \boldsymbol{\sigma}^{(s)}$ is measured instead of $\mathbf{n}^{(s)} \cdot \boldsymbol{\sigma}^{(s)}$. This results in a different detector backaction in the lower arm, given by

$$
\tilde{\mathcal{M}}^{(r)}=R^{-1}\left(\mathbf{n}^{(s)}\right) \sigma_{x}^{(s)} \tilde{M}^{(r)} \sigma_{x}^{(s)} R\left(\mathbf{n}^{(s)}\right),
$$

with

$$
\begin{aligned}
& \tilde{M}^{(0)}=\left(\begin{array}{cc}
1 & 0 \\
0 & \cos g-i \sin g \cos \theta^{(D)}
\end{array}\right)=M^{(0) \dagger}, \\
& \tilde{M}^{(1)}=\left(\begin{array}{cc}
0 & 0 \\
0 & -i \sin g \sin \theta^{(D)} e^{-i \varphi^{(D)}}
\end{array}\right)=M^{(1) \dagger},
\end{aligned}
$$

and the rotation matrix $R\left(\mathbf{n}^{(s)}\right)$ defined in Eq. (25). The same applies to the last postselected projective measurement, which is implemented when $g=\theta^{(D)}=-\varphi^{(D)}=\pi / 2$. is

The particle state just before passing the last beam splitter

$$
|\Psi\rangle=\frac{1}{\sqrt{2}} \sum_{\left\{r_{k}\right\}} \delta_{r_{N+1}, 0}\left|\psi_{0}\right\rangle \prod_{k=1}^{N+1}\left|r_{k}\right\rangle_{D_{k}}\left[\left\langle\psi_{0}\left|\mathcal{M}_{N}^{\left(r_{N}\right)} \cdots \mathcal{M}_{1}^{\left(r_{1}\right)}\right| \psi_{0}\right\rangle|+1\rangle_{a}+\left\langle\psi_{0}\left|\sigma_{x}^{(s)}\left(\mathbf{n}_{0}\right) \tilde{\mathcal{M}}_{N}^{\left(r_{N}\right)} \cdots \tilde{\mathcal{M}}_{1}^{\left(r_{1}\right)} \sigma_{x}^{(s)}\left(\mathbf{n}_{0}\right)\right| \psi_{0}\right\rangle|-1\rangle_{a}\right]
$$

where

$$
\sigma_{x}^{(s)}\left(\mathbf{n}_{0}\right)=R^{-1}\left(\mathbf{n}_{0}\right) \sigma_{x}^{(s)} R\left(\mathbf{n}_{0}\right)
$$

is the "FLIP" operator applied twice in the lower arm. Below we prove that

$$
\begin{aligned}
& \left\langle\psi_{0}\left|\sigma_{x}^{(s)}\left(\mathbf{n}_{0}\right) \tilde{\mathcal{M}}_{N}^{\left(r_{N}\right)} \cdots \tilde{\mathcal{M}}_{1}^{\left(r_{1}\right)} \sigma_{x}^{(s)}\left(\mathbf{n}_{0}\right)\right| \psi_{0}\right\rangle \\
& =\left\langle\psi_{0}\left|\mathcal{M}_{N}^{\left(r_{N}\right)} \cdots \mathcal{M}_{1}^{\left(r_{1}\right)}\right| \psi_{0}\right\rangle^{*}
\end{aligned}
$$

Using this identity, Eq. (63) for the intensities at the interferometer's exits immediately follows.

Proof of Eq. (C7). Note several identities. First,

$$
\begin{aligned}
& \left\langle\psi_{0}\left|\mathcal{M}_{N}^{\left(r_{N}\right)} \cdots \mathcal{M}_{1}^{\left(r_{1}\right)}\right| \psi_{0}\right\rangle \\
& \quad=\left(\begin{array}{ll}
1 & 0) \delta R M^{\left(r_{N}\right)} \cdots \delta R M^{\left(r_{1}\right)} \delta R\left(\begin{array}{l}
1 \\
0
\end{array}\right),
\end{array}\right.
\end{aligned}
$$

which follows from definitions of $\left|\psi_{0}\right\rangle$ in Eq. (32), $\mathcal{M}_{k}^{\left(r_{k}\right)}$ in Eq. (28), and $\delta R$ in Eq. (39). Second,

$$
\sigma_{x}^{(s)} \delta R \sigma_{x}^{(s)}=\exp \left(-\frac{2 \pi i d}{N+1}\right) \sigma_{z}^{(s)} \delta R^{\dagger} \sigma_{z}^{(s)}
$$

Finally,

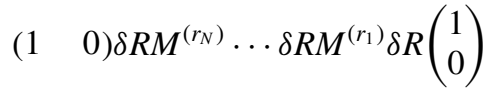

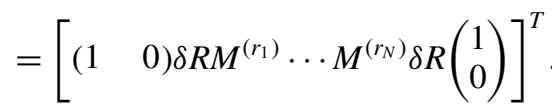

Using Eq. (C9), we show that

$$
\begin{aligned}
& \left\langle\psi_{0}\left|\sigma_{x}^{(s)}\left(\mathbf{n}_{0}\right) \tilde{\mathcal{M}}_{N}^{\left(r_{N}\right)} \cdots \tilde{\mathcal{M}}_{1}^{\left(r_{1}\right)} \sigma_{x}^{(s)}\left(\mathbf{n}_{0}\right)\right| \psi_{0}\right\rangle \\
& =\left(\begin{array}{ll}
1 & 0
\end{array}\right) \sigma_{x}^{(s)} \delta R \sigma_{x}^{(s)} \tilde{M}^{\left(r_{N}\right)} \cdots \tilde{M}^{\left(r_{1}\right)} \sigma_{x}^{(s)} \delta R \sigma_{x}^{(s)}\left(\begin{array}{l}
1 \\
0
\end{array}\right) \\
& =\left(\begin{array}{ll}
1 & 0
\end{array}\right) \sigma_{z}^{(s)} \delta R^{\dagger} \sigma_{z}^{(s)} \tilde{M}^{\left(r_{N}\right)} \cdots \tilde{M}^{\left(r_{1}\right)} \sigma_{z}^{(s)} \delta R^{\dagger} \sigma_{z}^{(s)}\left(\begin{array}{l}
1 \\
0
\end{array}\right) .
\end{aligned}
$$

Since $\sigma_{z}^{(s)} \tilde{M}^{(r)} \sigma_{z}^{(s)}=\tilde{M}^{(r)}$ and $\left(\begin{array}{ll}1 & 0\end{array}\right) \sigma_{z}^{(s)}=\left(\begin{array}{ll}1 & 0\end{array}\right)$,

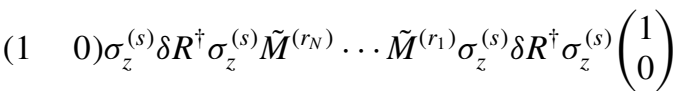

$$
\begin{aligned}
& =\left(\begin{array}{ll}
1 & 0
\end{array}\right) \delta R^{\dagger} \tilde{M}^{\left(r_{N}\right)} \cdots \delta R^{\dagger} \tilde{M}^{\left(r_{1}\right)} \delta R^{\dagger}\left(\begin{array}{l}
1 \\
0
\end{array}\right) .
\end{aligned}
$$


Using $\tilde{M}^{(r)}=M^{(r) \dagger}$ together with Eqs. (C8) and (C10), we show that

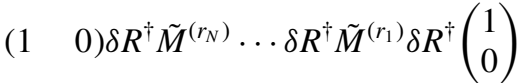

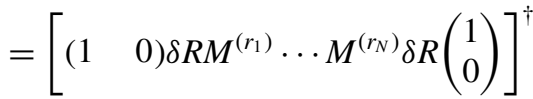

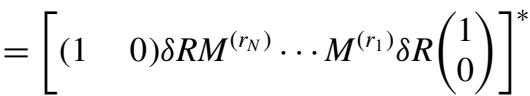

$$
\begin{aligned}
& =\left\langle\psi_{0}\left|\mathcal{M}_{N}^{\left(r_{N}\right)} \cdots \mathcal{M}_{1}^{\left(r_{1}\right)}\right| \psi_{0}\right\rangle^{*},
\end{aligned}
$$

which proves Eq. (C7).
[1] E. Cohen, H. Larocque, F. Bouchard, F. Nejadsattari, Y. Gefen, and E. Karimi, Geometric phase from Aharonov-Bohm to Pancharatnam-Berry and beyond, Nat. Rev. Phys. 1, 437 (2019).

[2] M. V. Berry, Quantal phase factors accompanying adiabatic changes, Proc. R. Soc. A 392, 45 (1984).

[3] D. J. Thouless, M. Kohmoto, M. P. Nightingale, and M. den Nijs, Quantized Hall Conductance in a Two-Dimensional Periodic Potential, Phys. Rev. Lett. 49, 405 (1982).

[4] Q. Niu, D. J. Thouless, and Y.-S. Wu, Quantized Hall conductance as a topological invariant, Phys. Rev. B 31, 3372 (1985).

[5] M. Z. Hasan and C. L. Kane, Colloquium: Topological insulators, Rev. Mod. Phys. 82, 3045 (2010).

[6] Y. Zhang, Y.-W. Tan, H. L. Stormer, and P. Kim, Experimental observation of the quantum Hall effect and Berry's phase in graphene, Nature (London) 438, 201 (2005).

[7] K. S. Novoselov, E. McCann, S. V. Morozov, V. I. Fal'ko, M. I. Katsnelson, U. Zeitler, D. Jiang, F. Schedin, and A. K. Geim, Unconventional quantum Hall effect and Berry's phase of $2 \pi$ in bilayer graphene, Nat. Phys. 2, 177 (2006).

[8] J. Pachos, P. Zanardi, and M. Rasetti, Non-Abelian Berry connections for quantum computation, Phys. Rev. A 61, 010305(R) (1999).

[9] J. A. Jones, V. Vedral, A. Ekert, and G. Castagnoli, Geometric quantum computation using nuclear magnetic resonance, Nature (London) 403, 869 (2000).

[10] C. Nayak, S. H. Simon, A. Stern, M. Freedman, and S. Das Sarma, Non-Abelian anyons and topological quantum computation, Rev. Mod. Phys. 80, 1083 (2008).

[11] Y. Aharonov and J. Anandan, Phase Change During a Cyclic Quantum Evolution, Phys. Rev. Lett. 58, 1593 (1987).

[12] See also A. A. Wood, K. Streltsov, R. M. Goldblatt, M. B. Plenio, L. C. L. Hollenberg, R. E. Scholten, and A. M. Martin, Interplay between geometric and dynamic phases in a singlespin system, Phys. Rev. B 102, 125428 (2020).

[13] M. V. Berry and S. Klein, Geometric phases from stacks of crystal plates, J. Mod. Opt. 43, 165 (1996).

[14] P. Facchi, A. G. Klein, S. Pascazio, and L. S. Schulman, Berry phase from a quantum Zeno effect, Phys. Lett. A 257, 232 (1999).

[15] D. Chruscinski and A. Jamiolkowski, Geometric Phases in Classical and Quantum Mechanics, Progress in Mathematical Physics (Birkhäuser Basel, Basel, 2004), Vol. 36.

[16] S. Pancharatnam, Generalized theory of interference, and its applications, Proc. Indian Acad. Sci. A 44, 247 (1956).

[17] B. Tamir and E. Cohen, Introduction to weak measurements and weak values, Quanta 2, 7 (2013).

[18] B. E. Y. Svensson, Pedagogical review of quantum measurement theory with an emphasis on weak measurements, Quanta 2, 18 (2013).
[19] Y.-W. Cho, Y. Kim, Y.-H. Choi, Y.-S. Kim, S.-W. Han, S.-Y. Lee, S. Moon, and Y.-H. Kim, Emergence of the geometric phase from quantum measurement back-action, Nat. Phys. 15, 665 (2019).

[20] V. Gebhart, K. Snizhko, T. Wellens, A. Buchleitner, A. Romito, and Y. Gefen, Topological transition in measurement-induced geometric phases, Proc. Natl. Acad. Sci. USA 117, 5706 (2020).

[21] M. A. Nielsen and I. L. Chuang, Quantum Computation and Quantum Information (Cambridge University Press, Cambridge, 2010).

[22] H. M. Wiseman and G. J. Milburn, Quantum Measurement and Control (Cambridge University Press, Cambridge, 2010), p. 460.

[23] K. Jacobs, Quantum Measurement Theory and its Applications (Cambridge University Press, Cambridge, 2014).

[24] K. Snizhko, P. Kumar, N. Rao, and Y. Gefen, WeakMeasurement-Induced Asymmetric Dephasing: Manifestation of Intrinsic Measurement Chirality, Phys. Rev. Lett. 127, 170401 (2021).

[25] E. Buks, R. Schuster, M. Heiblum, D. Mahalu, and V. Umansky, Dephasing in electron interference by a 'which-path' detector, Nature (London) 391, 871 (1998).

[26] M. Goldstein and Y. Gefen, Suppression of Interference in Quantum Hall Mach-Zehnder Geometry by Upstream Neutral Modes, Phys. Rev. Lett. 117, 276804 (2016).

[27] R. S. Whitney and Y. Gefen, Berry Phase in a Nonisolated System, Phys. Rev. Lett. 90, 190402 (2003).

[28] R. S. Whitney, Y. Makhlin, A. Shnirman, and Y. Gefen, Geometric Nature of the Environment-Induced Berry Phase and Geometric Dephasing, Phys. Rev. Lett. 94, 070407 (2005).

[29] R. S. Whitney, Y. Makhlin, A. Shnirman, and Y. Gefen, Berry phase with environment: classical versus quantum, in Theory of Quantum Transport in Metallic and Hybrid Nanostructures, edited by A. Glatz, V. I. Kozub, and V. M. Vinokur, NATO Science Series, Vol. 230 (Springer, Dordrecht, 2006), pp. 9-23.

[30] S. Berger, M. Pechal, P. Kurpiers, A. A. Abdumalikov, C. Eichler, J. A. Mlynek, A. Shnirman, Y. Gefen, A. Wallraff, and S. Filipp, Measurement of geometric dephasing using a superconducting qubit, Nat. Commun. 6, 8757 (2015).

[31] K. Snizhko, R. Egger, and Y. Gefen, Non-Abelian Geometric Dephasing, Phys. Rev. Lett. 123, 060405 (2019).

[32] K. Snizhko, R. Egger, and Y. Gefen, Non-Abelian Berry phase for open quantum systems: Topological protection versus geometric dephasing, Phys. Rev. B 100, 085303 (2019).

[33] A. C. Elitzur and S. Dolev, Nonlocal effects of partial measurements and quantum erasure, Phys. Rev. A 63, 062109 (2001). 
[34] G. S. Paraoanu, Interaction-Free Measurements with Superconducting Qubits, Phys. Rev. Lett. 97, 180406 (2006).

[35] X.-Y. Xu, J.-S. Xu, C.-F. Li, Y. Zou, and G.-C. Guo, Experimental demonstration of nonlocal effects in the partial-collapse measurement and reversal process, Phys. Rev. A 83, 010101(R) (2011).

[36] P. Lewalle, A. Chantasri, and A. N. Jordan, Prediction and characterization of multiple extremal paths in con- tinuously monitored qubits, Phys. Rev. A 95, 042126 (2017).

[37] M. Naghiloo, D. Tan, P. M. Harrington, P. Lewalle, A. N. Jordan, and K. W. Murch, Quantum caustics in resonancefluorescence trajectories, Phys. Rev. A 96, 053807 (2017).

[38] P. Lewalle, J. Steinmetz, and A. N. Jordan, Chaos in continuously monitored quantum systems: An optimal-path approach, Phys. Rev. A 98, 012141 (2018). 